\title{
A Coupled Fluid-Structure Interaction Analysis of Solid Rocket Motor with Flexible Inhibitors
}

\author{
H. Q. Yang ${ }^{1}$ and Jeff West ${ }^{2}$ \\ CFD Research Corporation, Huntsville \\ Jacobs ESSSA Group, Huntsville, AL, USA \\ NASA MSFC, Huntsville, AL 35805, USA
}

\begin{abstract}
A capability to couple NASA production CFD code, Loci/CHEM, with CFDRC's structural finite element code, $\mathrm{CoBi}$, has been developed. This paper summarizes the efforts in applying the installed coupling software to demonstrate/investigate fluid-structure interaction (FSI) between pressure wave and flexible inhibitor inside reusable solid rocket motor (RSRM). First a unified governing equation for both fluid and structure is presented, then an Eulerian-Lagrangian framework is described to satisfy the interfacial continuity requirements. The features of fluid solver, Loci/CHEM and structural solver, CoBi, are discussed before the coupling methodology of the solvers is described.

The simulation uses production level CFD LES turbulence model with a grid resolution of 80 million cells. The flexible inhibitor is modeled with full 3D shell elements. Verifications against analytical solutions of structural model under steady uniform pressure condition and under dynamic condition of modal analysis show excellent agreements in terms of displacement distribution and eigen modal frequencies. The preliminary coupled result shows that due to acoustic coupling, the dynamics of one of the more flexible inhibitors shift from its first modal frequency to the first acoustic frequency of the solid rocket motor.
\end{abstract}

$C F D=$ computational fluid dynamics

\section{Nomenclature}

\section{Introduction}

$\mathrm{D}$

uring the development of Ares I and Ares V launch vehicles, potential coupling between thrust oscillations in the solid rocket motor (SRM) first stage and vibration modes in the launch vehicle was identified as the top risk in the Ares I program. The frequency of pressure pulses in the five-segment SRM is close to the natural frequency of the second longitudinal vibration mode of the complete launch vehicle. This creates the risk of a "pogo stick" resonant vibration, which leads to the concerns that the vibration could make it difficult for the astronaut to perform their tasks, including reading their flight displays. As the thrust oscillation comes mainly from solid rocket motor pressure oscillation, an accurate predictive capability of pressure oscillation features considering all the important driving physics is crucial in the development of NASA's new Space Launch System (SLS).

Vortices emitted by an obstacle such as an inhibitor have been identified as the driving acoustic and combustion instability sources that can lead to thrust oscillation from the SRM. Flexible inhibitors have been used in the Space Shuttle Reusable Solid Rocket Motor (RSRM) to control the burning of propellant as illustrated in Figure 1 [1]. The inhibitor is an insulating material bonded to part of the propellant that prevents the underlying surface from becoming hot enough to ignite. The RSRM has inhibitors on the flat, forward-facing ends of the propellant in each of the 3 joint slots, which are annular rings made of asbestos-silica-filled nitrile butadiene rubber (NBR). The inhibitors help fine-tune the burning surface area, and therefore the thrust performance, to satisfy Shuttle requirements [1].

\footnotetext{
${ }^{1}$ Chief Scientist, CFD Research Crop., 215 Wynn Drive, Huntsville, AL 35805, and Senior AIAA Member

${ }^{2}$ Team Lead, Fluid Dynamics Branch-ER42, George C. Marshall Space Flight Center, MSFC, AL 35812, AIAA Member
} 


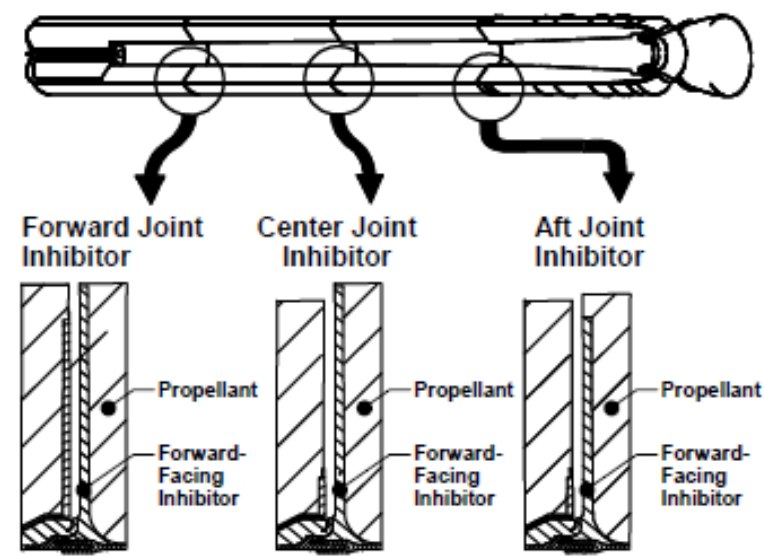

(a) RSRM propellant and inhibitor configuration for each field joint.

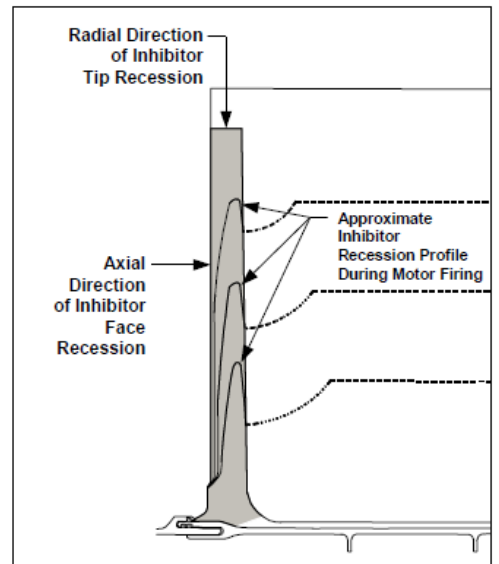

(b) General direction of RSRM inhibitor material recessions.

Figure 1.Flexible Inhibitors inside NASA's Space Shuttle Reusable Solid Rocket Motor [1]

The presence of an inhibitor can significantly affect the flow field in its immediate vicinity and some distance downstream, primarily through vortex shedding from the inhibitor tip. A number of cold-flow experiments and high fidelity numerical simulations have been performed to study the influence of inhibitors on pressure oscillations in rockets [2-4]. A recent study by Mastrangelo et al., [5] has identified the inhibitor as the main source of amplification of the pressure oscillation.

Since inhibitors often consist of relatively thin sheets of flexible materials, the difference in gas pressure on the forward- and aft-facing sides of the protruding portion of an inhibitor can cause it to bend in the direction of the flow. If the inhibitor is long and compliant enough, it can come into contact with the burning propellant surface downstream. Even if the inhibitor does not contact the propellant surface, it can be expected to oscillate about some equilibrium angle of deflection if viscoelastic damping in the solid and viscous damping from the fluid are small enough. It is very important to understand to the role of the flexible inhibitors in the coupling physics. For instance, does the inhibitor flexibility lead to the instability or does the flexibility of the inhibitor act as a means of controlling and reducing unstable modes in the SRM? For turbulent flow inside the SRM, the load on the inhibitor is generally 3D and chaotic and therefore very complex inhibitor motions are possible even if the material is isotropic and undamaged. It is important to understand not only the effect of inhibitor shape on the vortex shedding but also how the dynamically changing inhibitor geometry affects the flow.

Roach et al. [6,7] made attempts to study the flexible inhibitor interacting with a firing rocket motor using a quasi-static coupling. First, a static or time-dependent fluid-only computation was performed on the initial inhibitor geometry. The resulting pressure load was passed (through files) to a commercial Finite Element Method (FEM) solver, which determined the deformation under that prescribed load. The new geometry was passed (through files) back to the fluid solver, and the steady or unsteady flow was determined from the new geometry. Fiedler et al. [8] successfully computed the motion of a flexible inhibitor located in the core flow region aft of a joint slot in a fully coupled 3-D simulation. They demonstrated that an inhibitor flapped periodically with an angle of deflection ranging from 30 to $40 \mathrm{deg}$. Unfortunately, no information regarding such a flapping effect on the instability mechanism is available. Wasistho et al.[9] conducted a numerical study of 3-D flows past rigid and flexible inhibitors in the Space Shuttle Redesigned Solid Rocket Motor (RSRM). Only a section of the rocket near the center joint slot at 100 seconds after ignition was modeled using compressible dynamic Large Eddy Simulation (LES) for the fluid domain and an implicit finite element solver for the solid domain. Differences in the instantaneous and mean flow response to rigid and flexible inhibitors led to some useful hints regarding the design of inhibitor geometry and material.

As of this writing, no fully-coupled fluid-structure interaction simulation has been reported for production level (50 - 500 million cells) SRM study, and no mature tool to analyze and study the fluid-structure interaction in a solid rocket motor on a production simulation level exists. In a recent study, a comprehensive, fully coupled, high fidelity, user friendly multi-disciplinary simulation tool was developed by CFD Research Corp. (CFDRC) and Mississippi State University [10] to enable the investigation of the nonlinear interaction of flexible inhibitors with the vortical flow inside the SRM. The approach was to couple a NASA production CFD code, Loci/CHEM, for solid rocket 
motor ignition analysis (see the sample result in Figure 2a) with a DoD Open Source Finite Element Analysis code, $\mathrm{CoBi}$, developed at CFDRC for nonlinear large structural deformation (see example in Figure 2b).
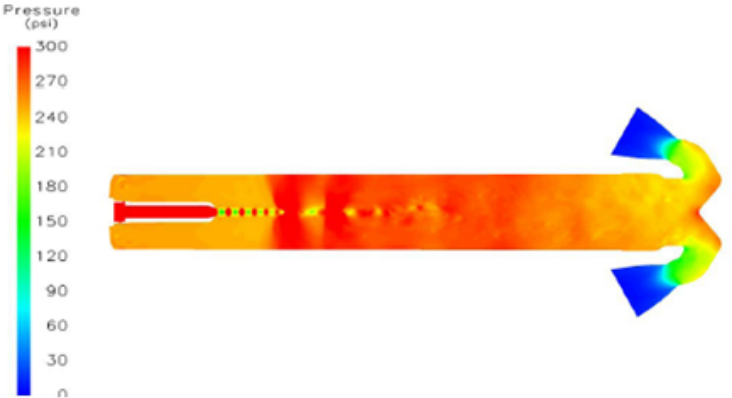

(a) Computed Pressure in side LAS Abort Motor by CFD Code Loci-CHEM

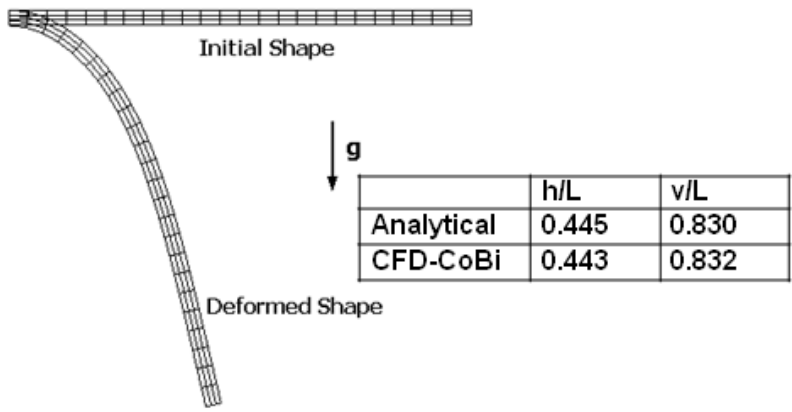

(b) Large Deformation of a Beam under Gravity Computed by the proposed FEM Structural Solver, CFD-CoBi

Figure 2. Capability demonstration each for CFD: Loci/CHEM and for Computational Structural Dynamics (CSD): CoBi.

The simulation tool with the above features is a highly valuable asset when the time comes to design a new motor or even when a modification is planned on existing geometries. The multi-disciplinary tool can improve pressure oscillation modeling fidelity and provide insight into nonlinear fluid-structure interaction leading to thrust oscillations in the preliminary design phase for future SRM for the Space Launch System. The present tool is also useful in identifying and predicting, as a first approximation and at a very early stage, the critical geometries and the time during firing at which thrust oscillations could occur.

\section{Mathematic Formulations}

Continuum Mechanics has been conventionally subdivided into solid mechanics and fluid mechanics. Even though both disciplines solve Newton's second law, they use different reference frames, different solution variables and different solution methods. For example, in structural dynamics, the dependent variables are displacements, and are typically formulated in a Lagrangian frame. These displacements are solved by Finite Element Method (FEM). In fluid dynamics, the dependent variables are velocities and are typically formulated in Eulerian frame and solved using the Finite Volume Method (FVM) or Finite Difference Method (FDM). Some of the characteristics of fluid dynamics and structural dynamics solvers are listed in Table 1.

As a result, problems involving interaction between fluid flow and solid deformation, with the examples of an aircraft body and wing, wind loaded structure and heat exchange, are generally treated separately and in a decoupled manner.There is, however, a wide range of problems that require simultaneous solution of fluid flow and solid body deformation, and thus require a uniform approach. This section will first present a unified formulation for both fluid and structure, and then describe the fluid and structural solvers used in this study. Finally the coupling methodology of the solvers will be discussed.

Table 1. Comparison of fluid dynamics and structural dynamics solutions 


\section{Fluid Dynamics}

\section{Reference Frame}

Discretization

Solution Variables

Equation Type

Unknown Location

Expressionfor

Acceleration, a

Solvers

\section{Constitutive}

Relation
Eulerian

Fin ite Volume Method

Velocity $v_{x}, v_{y}, v_{z}$

Parabolic or Elliptic

Cell Center

$\frac{d \vec{V}}{d t}=\frac{\partial \vec{V}}{\partial t}+\frac{\partial \vec{V}}{\partial \vec{x}}-\frac{\partial \vec{x}}{\partial t}=\frac{\partial \vec{V}}{\partial t}+(\vec{V} \cdot V) \vec{V}$

Loci/CHEM, Loci/STREAM

Proportional to Velocity

Gradient

\section{Structural Dynamics}

Lagrangian

Finite Element Method

Displacement $\mathrm{u}_{\mathrm{x}}, \mathrm{u}_{\mathrm{y}}, \mathrm{u}_{\mathrm{z}}$

Hyperbolic
Cell Node

$$
\frac{d \vec{V}}{d t}=\frac{\partial \vec{V}}{\partial t}
$$

Nastran

Proportional to

Displacement Gradient

\section{A Unified Governing Equation}

Regardless of the solution method (FEM, FVM, or FDM), the solid body and fluid mechanics actually share the same governing equations, and differ only in constitute relations. The governing equation for both a fluid and a solid are the momentum equations of Newton's law:

$$
\rho \dot{v}_{i}=\sigma_{i j, j}+f_{i}
$$

where $\rho$ is the density, $v_{i}$ is the velocity, $\sigma_{i j}$ is the stress tensor, $f_{i}$ is the internal body force, a superscript dot designates a total derivative, a comma a partial derivative with respect to the following variable. Repeated indices denote summation over the appropriate range. To close the system in Equation (1), the information about the response of particular material to applied force is necessary. Here we will take a compressible gas and elastic solid as example of fluid and solid materials, respectively.

\section{For fluid dynamics:}

The Equation of State is:

$$
\rho=\rho(p, T)
$$

where $\mathrm{p}$ is pressure and $\mathrm{T}$ is temperature. For an incompressible fluid :

$$
\rho=\text { constant }
$$

and for an ideal gas:

$$
\rho=\frac{\mathrm{p}}{\mathrm{RT}}
$$

The constitutive relation between stress and rate of deformation for fluids is given by Stokes' law: 


$$
\sigma_{i j}=\mu\left(v_{i, j}+v_{j, i}\right)-\frac{2}{3} \mu \delta_{i j} v_{k, k}-p \delta_{i j}
$$

where $\mu$ is the dynamic viscosity.

For an elastic solid,

The constitutive relationship is given by Hooke's law:

$$
\sigma_{i j}=\eta\left(u_{i, j}+u_{j, i}\right)+\lambda \delta_{i j} u_{k, k}
$$

where $\eta$ and $\lambda$ are the Lame constants, $\mathrm{u}_{\mathrm{i}}$ is the displacement vector and

$$
\mathrm{v}_{\mathrm{i}}=\dot{\mathrm{u}}_{\mathrm{i}}
$$

Note that in the fluid the stress is expressed in terms of velocity, whereas, in the solid the stress is expressed in terms of displacement.

\section{B. Consistent Interface Boundary Condition}

As for interface boundary conditions, it is required that displacement, velocity and stresses are continuous, i.e.

$$
\begin{gathered}
\left(\mathrm{u}_{\mathrm{i}}\right)_{\mathrm{s}}=\left(\mathrm{u}_{\mathrm{i}}\right)_{\mathrm{f}} \\
\left(\mathrm{v}_{\mathrm{i}}\right)_{\mathrm{s}}=\left(\mathrm{v}_{\mathrm{i}}\right)_{\mathrm{s}} \\
\left(\left(\sigma_{i j}\right)_{n}\right)_{s}=\left(\left(\sigma_{i j}\right)_{n}\right)_{f} \\
\left(\left(\sigma_{i j}\right)_{\tau}\right)_{s}=\left(\left(\sigma_{i j}\right)_{\tau}\right)_{f}
\end{gathered}
$$

with subscript $\mathrm{s}$ and $\mathrm{f}$ representing solid and fluid domains and $\mathrm{n}$ and $\tau$ are the normal and tangential directions of with respect to the interface.

\section{Reference Frame and Mesh Systems}

Before an equation is discretized, it is important to select the appropriate reference frame. In classic solid mechanics, the dynamics equation is formulated in a Lagrangian frame, where:

$$
\rho \dot{\mathrm{v}}_{\mathrm{i}}=\rho \frac{\mathrm{dv}_{\mathrm{i}}}{\mathrm{dt}}
$$

Here one moves with or follows the structure. In classic fluid dynamics, the conservation equation is formulated in an Eulerian frame, where:

$$
\rho \dot{v}_{i}=\rho \frac{\partial v_{i}}{\partial t}+v_{i, j} v_{j}
$$

It is the second nonlinear term that has given rise to many difficulties in fluid dynamics. However, in the fluid dynamics approach, the Eulerian frame is necessary. To distinguish the difference between the two reference frames, we denote the space (Eulerian) coordinate by $\mathrm{x}_{\mathrm{i}}$, the material (Lagrangian) coordinate by $\mathrm{X}_{\mathrm{i}}$, and mesh coordinate by $\chi_{\mathrm{i}}$. Then if our mesh is given by 


$$
\chi_{\mathrm{i}}=\mathrm{x}_{\mathrm{i}}
$$

we have identified an Eulerian mesh and when

$$
\chi_{\mathrm{i}}=\mathrm{X}_{\mathrm{i}}
$$

is used, we are identifying a Lagrangian mesh.

\section{Eulerian-Lagrangian (Fluid) - Lagrangian (Solid) Approach}

Since structural dynamics equations are formulated in Lagrangian frame whereas the fluid dynamics equations are formulated in Eulerian frame, direct coupling of two solvers could lead to overlapped meshes or holes in the solution domain. This is illustrated in Figure 3.
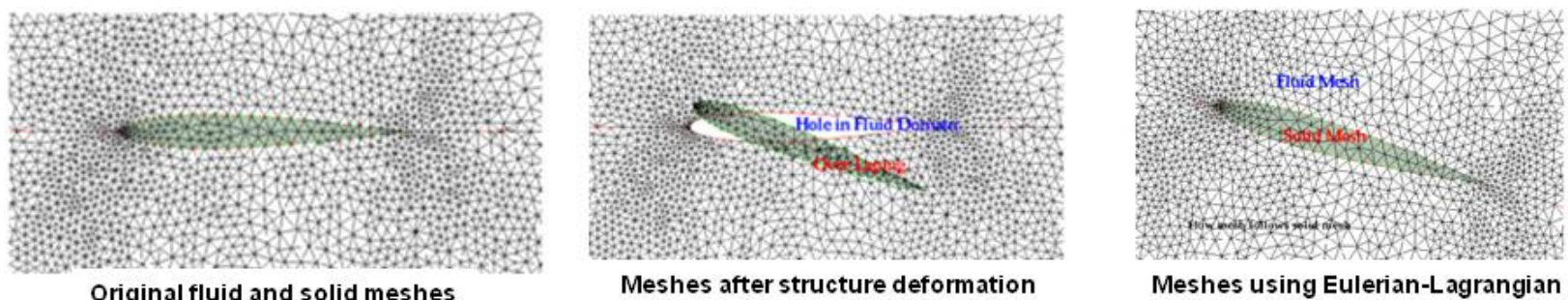

Meshes after structure deformation

Meshes using Eulerian-Lagrangian

\section{Figure 3.Mesh deformation for Eulerian and Eulerian-Lagrangian approach}

The critical requirement for reference frames is to preserve fluid-structure interface and prevent cutting and crossing. This will ensure that correct interface conditions are applied. The Eulerian-Lagrangian (fluid) Lagrangian (solid) approach has the above properties as seen from Figure 3. Within this formulation, a mesh velocity is introduced and the momentum equation becomes:

$$
\rho \frac{\partial v_{i}}{\partial t}+\left(v_{j}-v_{G j}\right) v_{i, j}=\sigma_{i j, j}+f_{i}
$$

Here we set the mesh velocity as:

$$
\begin{gathered}
\mathrm{v}_{\mathrm{Gj}}=\mathrm{v}_{\mathrm{j}} \text { in the solid } \\
\left(\mathrm{v}_{\mathrm{Gj}}\right)_{\mathrm{fs}}=\left(\mathrm{v}_{\mathrm{j}}\right)_{\mathrm{fs}} \text { at the solid-fluid interface }
\end{gathered}
$$

To accomplish the Eulerian-Lagrangian framework for the fluid, a volumetric mesh deformation inside the fluid mesh is required.

\section{Solution of Mathematic Equations}

\section{A. Fluid Dynamics Solver}

A fully coupled fluid-structure interaction approach requires solvers for both fluid and structural phases. A NASA production CFD code for solid rocket motor, Loci/CHEM, is used to solve the compressible fluid dynamics. The Loci/CHEM code is a modern multiphysics simulation code that is capable of modeling chemically reacting multiphase high- and low-speed flows. CHEM, which was developed at Mississippi State University, is written in the Loci framework [11] and is a Reynolds-averaged Navier-Stokes, finite-volume flow solver for generalized or arbitrary polyhedral mesh. Loci is a novel software framework that has been applied to the simulations of nonequilibrium flows. The Loci system uses a rule-based approach to automatically assemble the numerical simulation 
components into a working solver. This technique enhances the flexibility of simulation tools, reducing the complexity of CFD software induced by various boundary conditions, complex geometries, and varied physical models. Loci plays a central role in building flexible goal-adaptive algorithms that can quickly match numerical techniques with various physical modeling requirements. Loci/CHEM has also a volume mesh deformation module.

Loci/CHEM [12] uses density-based algorithms and employs high-resolution approximate Riemann solvers to solve finite-rate chemically reacting viscous turbulent flows. It supports adaptive mesh refinement, simulations of complex Equations of State including cryogenic fluids, multiphase simulations of dispersed particulates using both Lagrangian and Eulerian approaches, conjugate heat transfer through solids [13], and non-gray radiative transfer associated with gas and particulate phases. It supports multiple two equation RANS turbulence models including Wilcox's k- $\omega$ model; Menter's baseline model; and Menter's shear stress transport model (SST) [14]. For unsteady flow problems such as the present rocket motor acoustic waves, Loci/CHEM has hybrid RANS/LES turbulence model treatments that include high speed compressibility corrections. The Loci/CHEM CFD code is a library of Loci rules (fine-grained components), and provides primitives for generalized mesh, including metrics; operators, such as gradient; chemically reacting physics models, such as equations of state, inviscid flux functions, and transport functions (viscosity, conduction, and diffusion); a variety of time and space integration methods; linear system solvers; and more. Moreover, it is a library of reusable components that can be dynamically reconfigured to solve a variety of problems involving generalized mesh by changing the given fact database, adding rules, or changing the query.

Using the sophisticated automatic parallelization framework of Loci, Loci/CHEM has demonstrated scalability to problems in size exceeding five hundred million cells and production scalability to four thousand processors.

\section{B. Nonlinear Structure Dynamics Solver}

The structural dynamics solver used in this study, CoBi, is a DoD Open Source code developed at CFDRC. CoBi discretizes the dynamics equation (1) using the Finite Element Method as:

$$
\left[M_{s}\right] \frac{\partial\left\{v_{s}\right\}}{\partial t}+[D]\left\{v_{s}\right\}+K\left\{u_{s}\right\}=\left\{f_{s}\right\}
$$

Where $\mathrm{M}_{\mathrm{s}}, \mathrm{v}_{\mathrm{s}}, \mathrm{D}, \mathrm{K}, \mathrm{u}_{\mathrm{s}}, \mathrm{f}_{\mathrm{s}}$ denote respectively the mass matrix, the velocity, the damping matrix, the stiffness matrix, the displacements and the loads.

The main capabilities of the solver include:

- triangular, quadrilateral, tetrahedral, prismatic, or brick elements

- linear or high-order isoparametric elements;

- small or large deformations;

- elastic or plastic stresses;

- isotropic or anisotropic materials;

- thin to thick shell/plate elements;

- modal analysis and eigenvalue solutions; and

- steady and dynamic analysis.

To consider the large deformation of the inhibitor, the nonlinear strain due to large deformations will be taken into account. The nonlinear structural effect can shed light on the complex coupling of acoustics with structural vibrations, not available in classical linear analysis. Typically, for the elastic solid, the linear constitutive relationship is given by Hooke's law (Equation 6):

$$
\sigma_{i j}=\eta\left(u_{i, j}+u_{j, i}\right)+\lambda \delta_{i j} u_{k, k}
$$

where $\eta$ and $\lambda$ are the Lame constants and $\mathrm{u}_{\mathrm{i}}$ is the displacement vector. A comma denotes a partial derivative with respect to the following variable. To include the nonlinear geometrical contribution, additional terms have to be added such that:

$$
\sigma_{i j}=\eta\left(\left[\left(u_{i, j}+u_{j, i}+u_{k, j} u_{j, k}\right)+\lambda \delta_{i j}\left(u_{k, k}+u_{m, k} u_{m, k}\right)\right]\right.
$$


To expand the above expression, for example, the strain-displacement for $\varepsilon_{\mathrm{x}}$ and $\varepsilon_{\mathrm{xy}}$ can be written as:

$$
\begin{gathered}
\varepsilon_{\mathrm{x}}=\frac{\partial \mathrm{u}}{\partial \mathrm{x}}+\frac{1}{2}\left[\left(\frac{\partial \mathrm{u}}{\partial \mathrm{x}}\right)^{2}+\left(\frac{\partial \mathrm{v}}{\partial \mathrm{x}}\right)^{2}+\left(\frac{\partial \mathrm{w}}{\partial \mathrm{x}}\right)^{2}\right] \\
\varepsilon_{\mathrm{xy}}=\frac{\partial \mathrm{u}}{\partial \mathrm{x}}+\frac{\partial \mathrm{v}}{\partial \mathrm{y}}+\left[\frac{\partial \mathrm{u}}{\partial \mathrm{x}} \cdot \frac{\partial \mathrm{u}}{\partial \mathrm{y}}+\frac{\partial \mathrm{v}}{\partial \mathrm{x}} \cdot \frac{\partial \mathrm{v}}{\partial \mathrm{y}}+\frac{\partial \mathrm{w}}{\partial \mathrm{x}} \cdot \frac{\partial \mathrm{w}}{\partial \mathrm{y}}\right]
\end{gathered}
$$

Similar expressions can be written for the other strain components. The nonlinear terms in the above expression are due to geometrically large deformations. As such, the structural dynamics equation will no longer be linear. In this study, the final nonlinear equation is solved by Newton's method in FEM module, where the stiffness matrix will be represented as:

$$
[\mathrm{k}]=\left[\mathrm{k}_{\mathrm{o}}\right]+\left[\mathrm{k}_{\sigma}\right]+\left[\mathrm{k}_{\mathrm{L}}\right]
$$

in which: $\left[\mathrm{k}_{\mathrm{o}}\right]$ is the linear small displacement stiffness matrix;

$\left[\mathrm{k}_{\sigma}\right]$ is a symmetric matrix dependent on the stress level;

$\left[\mathrm{k}_{\mathrm{L}}\right]$ is known as the initial displacement matrix.

\section{Fluid-Structure Coupling and Data Exchange}

The coupling of the structural dynamics solver, CoBi, with the aerodynamics solver, Loci/CHEM, is accomplished through the boundary conditions across the solid-fluid interface. The boundary conditions require the continuity of interface displacement and velocity, and the continuity of normal and tangential forces, as stated in Equations (8-10). Since each solver (structural and fluid) employ a dual-time method, the pseudo-time iteration is applied to ensure strong coupling. Within each real-time step, the flow solution and the structural solution are repeatedly advanced by several pseudo-time steps followed by an update of the aerodynamic forces and mesh deformations. This procedure is repeated until the flow and displacements are converged before proceeding to the next real-time step. This modular treatment allows one to apply well-established and optimized methods for the flow and the structure, respectively.

In practical applications, the CFD model may use a much finer discretization than does the CSD model. As a result, the mesh used for discretization of the structural mode shapes does not coincide with the flow mesh as shown in Figure 4. In our current study, aerodynamic loads are obtained on the body-matched flow mesh and will be projected onto the structural mesh. Deformations obtained on the structural mesh have to be transferred to the flow mesh. Both transformations have to satisfy the requirements of conservation of work and energy. 


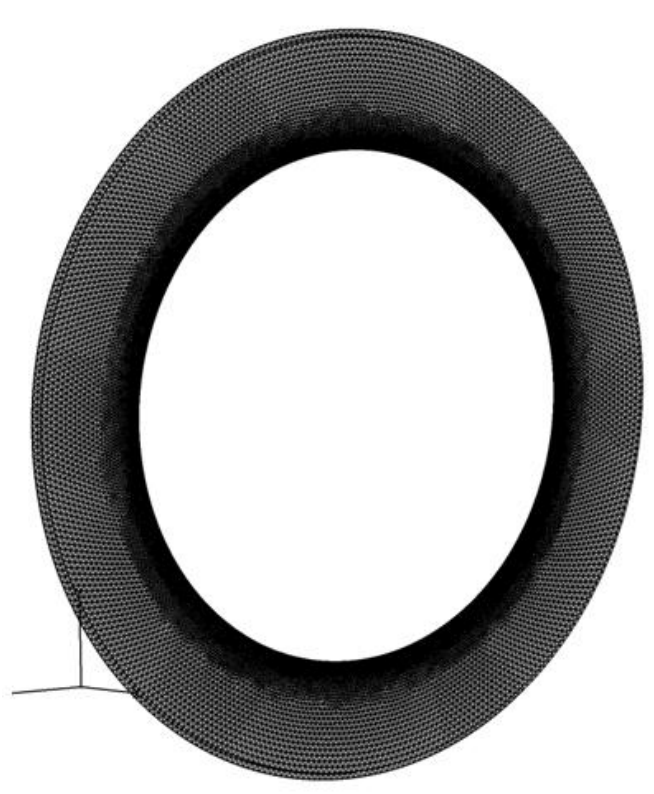

Fluid grid with triangular faces on interface of inhibitor

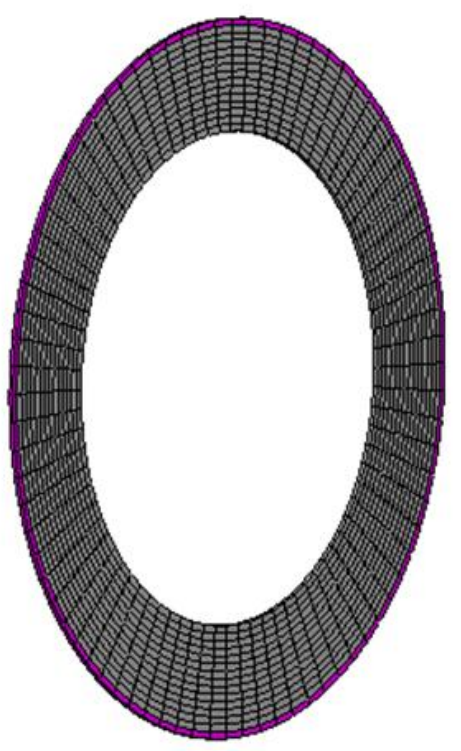

Structural grid with quadrilateral faces on interface of inhibitor

Figure 4.Processing of nonmatching interface from solid and structure.

The principle of virtual work has been employed to ensure conservation. For a linear transformation, let the displacements $\left\{\mathrm{x}_{\mathrm{a}}\right\}$ of the aerodynamic mesh to be expressed in terms of the structural mesh displacements $\left\{\mathrm{x}_{\mathrm{s}}\right\}$ using a transformation matrix $[\mathrm{G}]$ :

$$
\left\{x_{a}\right\}=[G]\left\{x_{s}\right\}
$$

then the requirement for conservation leads to a corresponding matrix for the transformation of forces:

$$
\left\{f_{s}\right\}^{T}\left\{\Delta x_{s}\right\}=\left\{f_{a}\right\}^{T}\left\{\Delta x_{a}\right\}=\left\{f_{a}\right\}^{T}[G]\left\{\Delta x_{s}\right\}
$$

which leads to:

$$
\left\{\Delta f_{s}\right\}^{T}=[G]^{T}\left\{\Delta f_{a}\right\}
$$

In this way, the global conservation of work can be satisfied regardless of the method that is used to obtain the transformation matrix. During the current coupling procedure, Loci/CHEM provides traction vectors at cell centers which are then interpolated to nodal forces on an interface mesh in the CoBi model through the above transformation.

\section{Model Description}

We now apply the tightly-coupled fluid-structure interaction capabilities to simulate the flow and structural response inside the RSRM with flexible inhibitors separating the propellant sections. The computational model is a RSRM at 80 seconds after ignition as shown in Figure 4. The model has propellant grain surface, solid walls, and exit boundaries. All the geometrical model, boundary condition, and initial condition were based on the work by Phil Davis of NASA MSFC at ER42 [15].

\section{A. Fluid Dynamics Mesh}

An initial unstructured hybrid prism/pyramid/tet computational mesh comprised of $62.5 \mathrm{M}$ cells was generated for this application. The solid rocket motor was meshed using 1 inch surface spacing for the grain and walls. Finer 
spacing was used at the inhibitors and coarse spacing was used downstream of the throat. An illustration of the cross-sectional cut through the computational mesh is shown in Figure 5.
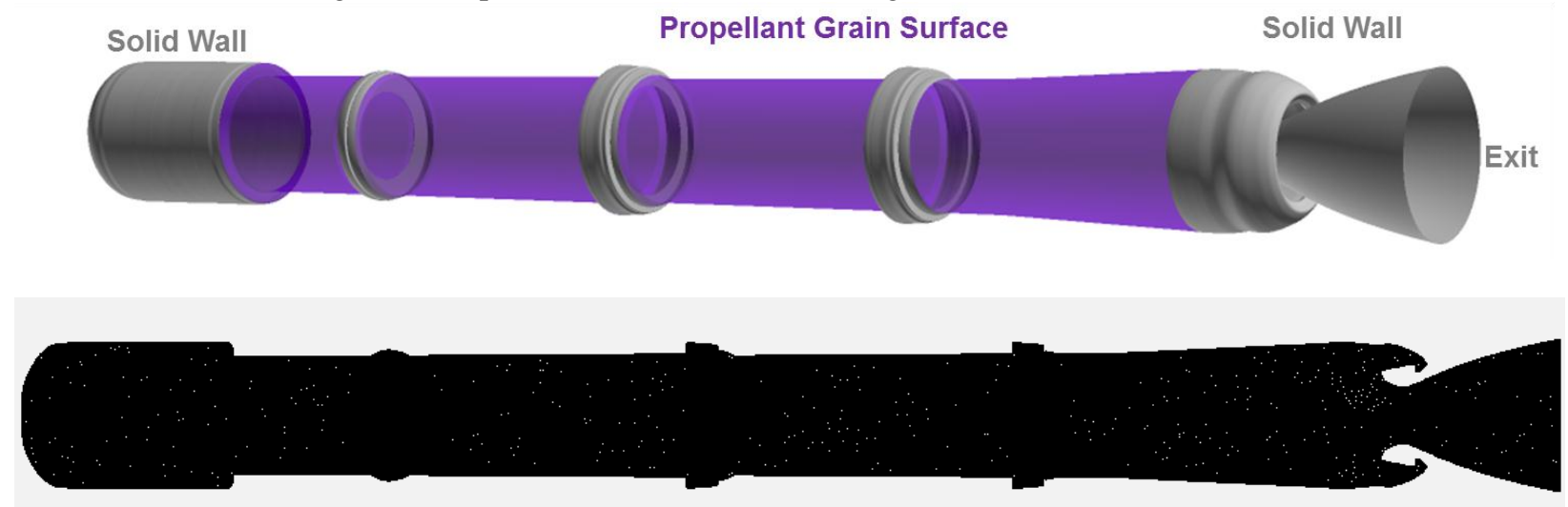

Figure 5.Geometry for reusable solid rocket motor (RSRM) problem: (Top) Illustration of propellant grain surface, solid wall, and exit boundaries; (Bottom) Cross-sectional cut of 3Dmesh.

To better capture and preserve vortices shed from the inhibitors, a new mesh was generated where cells were packed near the path of the vortex street from the inhibitors as shown in Figure 6. The new mesh keeps the same spacing on the grain and solid wall surfaces with a total cell count of 80 Million.

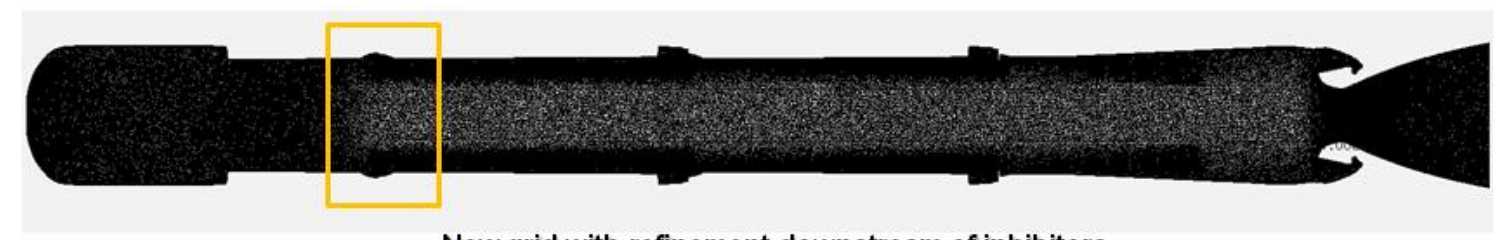

New grid with refinement downstream of inhibitors
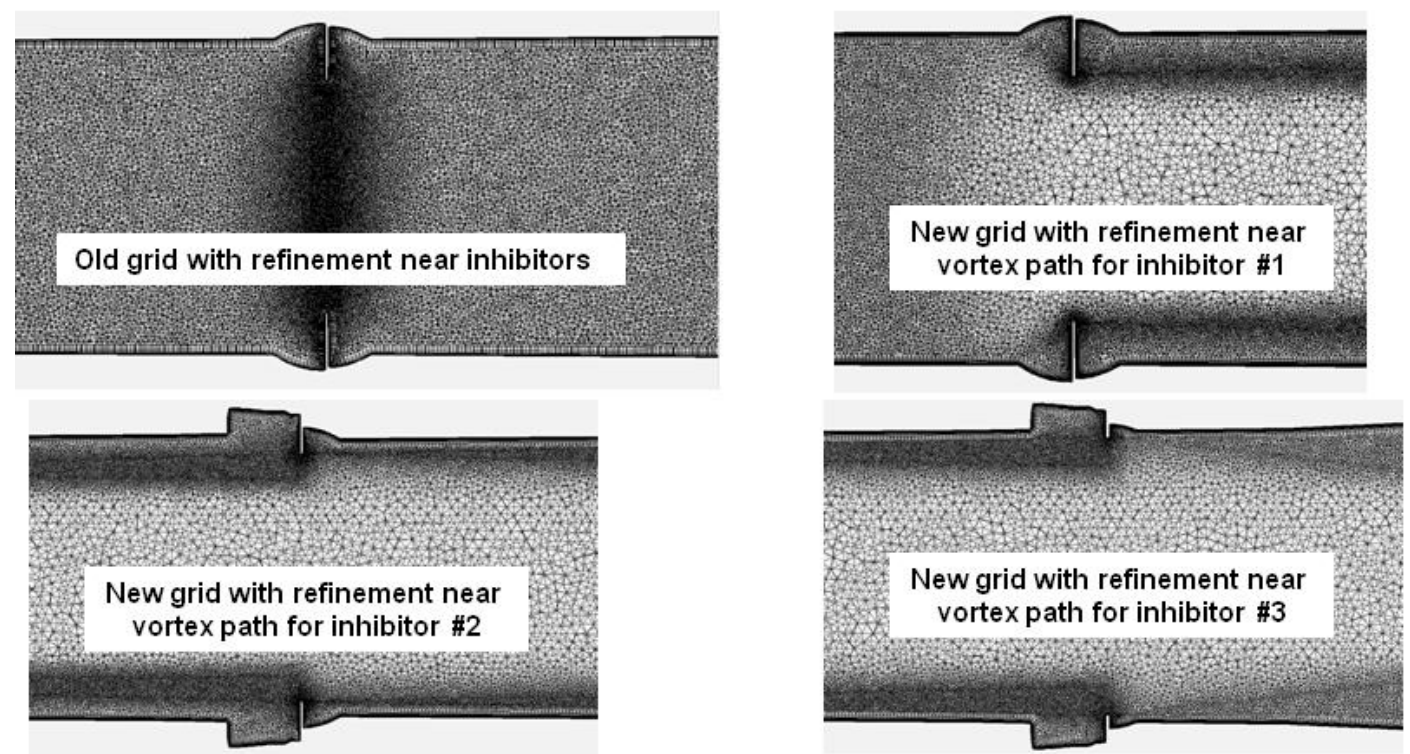

Figure 6.The refined mesh with packed cells near the vortex path for three inhibitors.

\section{B. Fluid Dynamics Solver Settings}

For the Loci/CHEM flow solution, $2^{\text {nd }}$ order spatial and temporal accuracy is employed, with the $2^{\text {nd }}$ order upwind inviscid flux and Venkatakrishnan limiter. A time step of $0.0001 \mathrm{~s}$ is used with 8 Newton iterations per time step, and urelax $=0.4$. Sutherland's law is applied as the laminar transport model, and Mentor shear stress transport 
(SST) turbulence model with multi-scale LES is employed. Direct Numerical Simulation (DNS) calculations are impractical for complex flows and RANS calculations are not strictly applicable to unsteady flows. The technique of combining traditional RANS with Large Eddy Simulation (LES) (hybrid RANS/LES) offers an affordable alternative. With hybrid RAN/LES, only the largest turbulent eddy structures that can be adequately resolved on a given mesh are simulated. The smallest remaining structures and the turbulence energy contained within them are then modeled using a sub mesh scale model. This technique allows a more appropriate representation of the unsteady turbulent fluctuations than RANS alone, and it is computationally feasible for the present unsteady flow with vortex shedding and breakdown.

A single phase equivalent gas model is used as the chemistry model for the RSRM gas [15]. An adiabatic, no-slip boundary condition is employed on all solid walls and a supersonic outflow condition is employed at the exit. On the propellant grain surfaces, gas at $3996 \mathrm{~K}$ is injected in the normal direction into the fluid domain with a mass flux determined from the steady burn-rate formula of:

$$
\dot{m}=\rho_{s} a P^{n}
$$

Here, $\rho_{\mathrm{s}}$ is propellant density at $1764 \mathrm{~kg} / \mathrm{m}^{3} ; a$ is the burning rate constant of 0.604 , and $\mathrm{n}=0.32$.

\section{Structural Dynamics Mesh}

The computational mesh for the finite element solution on the three flexible inhibitors is shown in Figure 7. All three inhibitor meshes are comprised of 80 cells in the circumferential direction, and 1 cell in the axial direction, with 14,10 , and 7 cells in the axial direction for the $1^{\text {st }}, 2^{\text {nd }}$, and $3^{\text {rd }}$ inhibitor, respectively. As the inhibitor is very thin, the problem will be solved using the shell element formulation. The above discretization yields 1120,800 and 560 shell elements for the $1^{\text {st }}, 2^{\text {nd }}$, and $3^{\text {rd }}$ inhibitor, respectively. Unlike the fluid mesh, where the smallest vortex could dominate the fluid physics such as in turbulent flow, in structural dynamics the first few modal deformations dominate the structural response. Due to this property, it is possible to use a much coarser mesh for the structural solution. The results for the modal analysis will be presented in Section 5.

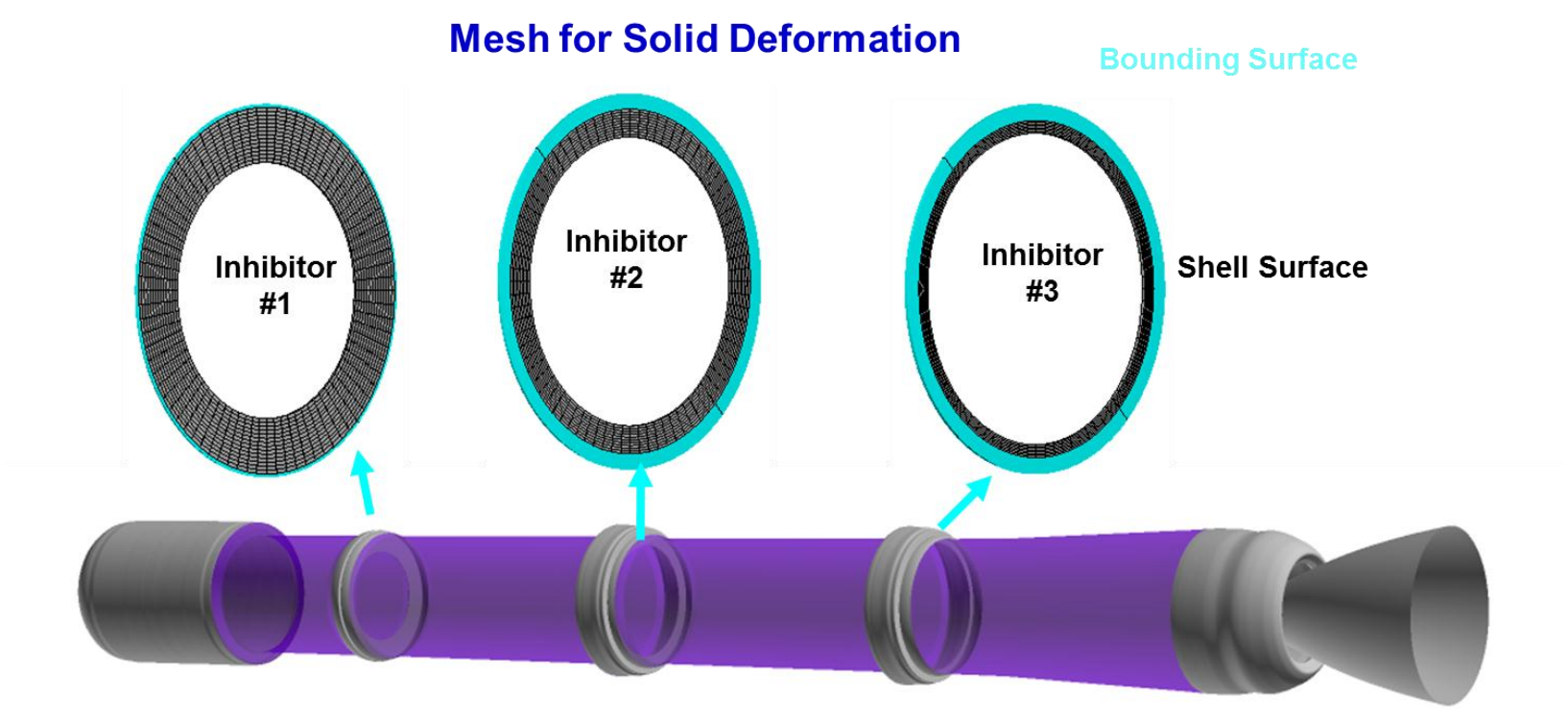

Figure 7.Geometry for RSRM with close-up on flexible inhibitors showing the portion of each inhibitor that is fixed (cyan) and the part that is flexible (black).

\section{Structural Dynamics Setting}

As can be seen in Figure 7, the bounding surfaces on all three inhibitors are fixed as zero displacement. The $1^{\text {st }}$ inhibitor thus has the largest surface area that is capable of deforming, and the $3^{\text {rd }}$ inhibitor has the smallest. A linear elasticity model is used for the solid material in which the elastic modulus $\mathrm{E}=1.8 \times 10^{8} \mathrm{~Pa}[6,7,9]$, Poisson ratio $\nu=0.49998$ and density $\rho=1,000 \mathrm{~kg} / \mathrm{m}^{3}$. Nonlinear large geometrical deformations are permitted. During the initial 
testing, it was found the above Young's modulus gives excessive deformation of the inhibitor, making a converged solution impossible. It was decided to use larger values of $E=1.4 \times 10^{9} \mathrm{~Pa}$, and $\rho=7,800 \mathrm{~kg} / \mathrm{m} 3$. As both Young's modulus and density are increased at the same ratio, this will ensure the same modal frequency of the inhibitors.

For the structural solver, a $2^{\text {nd }}$ order accurate temporal scheme of Newmark is used, with a time step the same as that in the CFD solver of $0.0001 \mathrm{~s}$ for all simulations.

\section{Results}

This section will discuss the results of coupled fluid-structure interaction in RSRM. First the results from the decoupled solutions will be presented and discussed.

\section{A. Fluid Solution With Rigid Inhibitor}

In preparation for the fully coupled fluid-structure interaction simulation, we first perform fluid-only simulations with rigid structures to allow the flow inside the solid rocket motor to develop to a nearly fully-developed state. The problem can be very stiff in the beginning, so we employ $1^{\text {st }}$ order schemes to establish the initial flow field. A representative instantaneous flow field from an initial run is shown in Figure 8. The instantaneous Mach number, vorticity and pressure fields are displayed at the top, middle, and bottom of the figure, respectively. As one can see from the Mach number contours, the flow is almost fully developed. The initial flow inside the RSRM is clearly vortex-dominated with heavy vortex shedding present at all of the rigid inhibitors, which locally produce lower pressures due to the vortices as observed just downstream of the first inhibitor. There is also a clear net pressure gradient axially toward the nozzle exit, which will ultimately act to bend the inhibitors in that direction during the coupled FSI simulations.

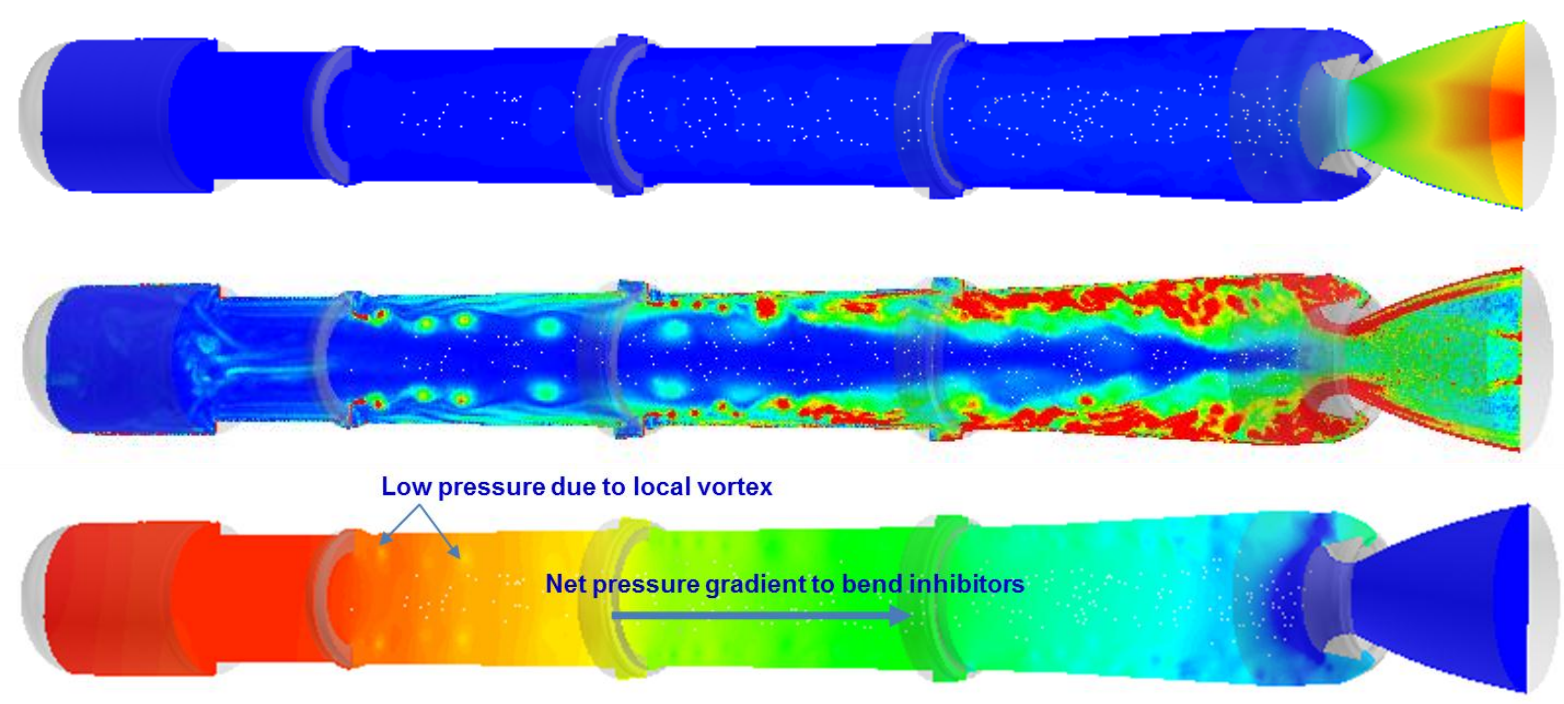

Figure 8.Initial transient CFD solution for RSRM with rigid inhibitors: (Top) Instantaneous Mach number field; (Middle) Instantaneous vorticity field; (Bottom) Instantaneous pressure field.

\section{B. Structural Solution Without Fluid Forcing}

The current CFD solver Loci/CHEM has been used at NASA MSFC for many years and it has been validated and verified for many different applications. On the other hand, the current structural solver, $\mathrm{CoBi}$, is not well known. To instill some confidence in the structural solver, we will first present two verification cases related to the inhibitor: the bending of a circular plate and the first four natural frequencies of the circular plate, as shown in Figure 9. In these two cases, analytical solutions are known and available. 

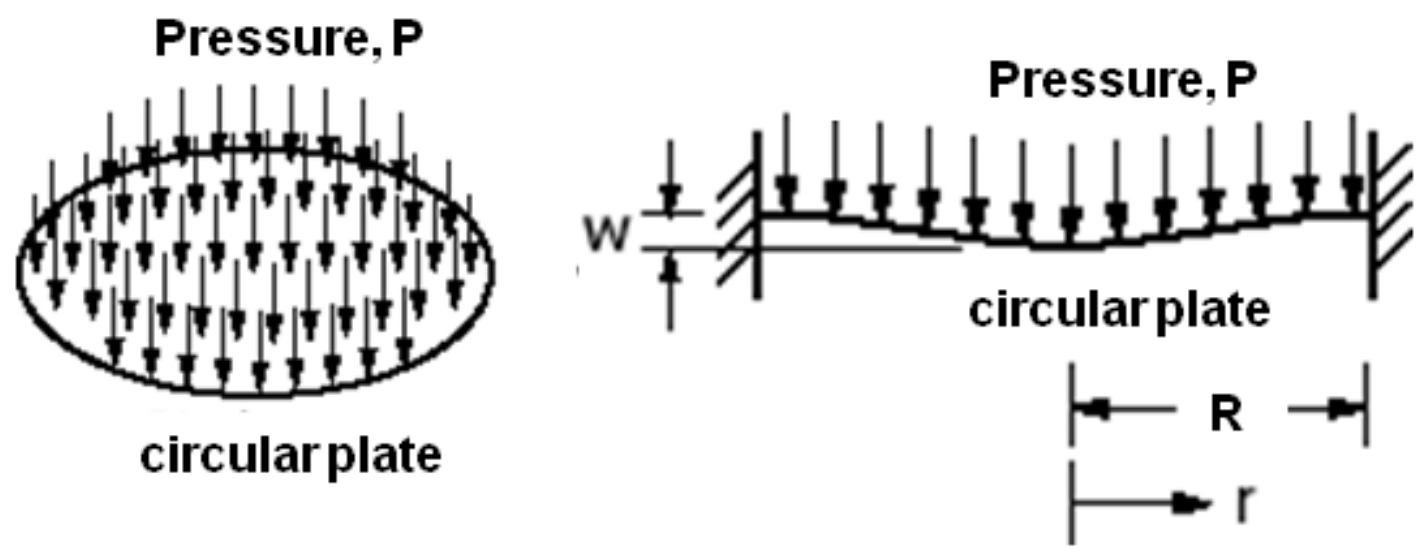

Figure 9.Bending of a circular plate under uniform pressure

The governing equation for the linear bending of the circular plate under uniform pressure force $\mathrm{P}$ is [16]

$$
\nabla^{4} w=\left(\frac{d^{2}}{d r^{2}}+\frac{1}{r} \frac{d}{d r}\right)\left(\frac{d^{2}}{d r^{2}}+\frac{1}{r} \frac{d}{d r}\right) w=\frac{P}{D} q
$$

Where $\mathrm{w}$ is the deflection of the plate, $\mathrm{D}$ is the bending rigidity of the plate,

$$
D=\frac{E t^{3}}{12\left(1-v^{2}\right)}
$$

The parameters $t$ and $v$ are the thickness and Poisson's ratio of the plate material, respectively. With a clamped edge boundary:

$$
w=0 ; \frac{d w}{d r}=0, \quad \text { at } \mathrm{r}=\mathrm{R}
$$

One can find the analytical solution in the form of:

$$
w(r)=\frac{P R^{4}}{64 D}\left[1-\left(\frac{r}{a}\right)^{2}\right]^{2}
$$

The verification model has the similar geometrical and mesh sizes as the first inhibitor as shown in Figure 10 . The computed plate deflection from CoBi shell element solution is compared with the above analytical solution in Figure 11. As one can see both solutions are essentially identical. 


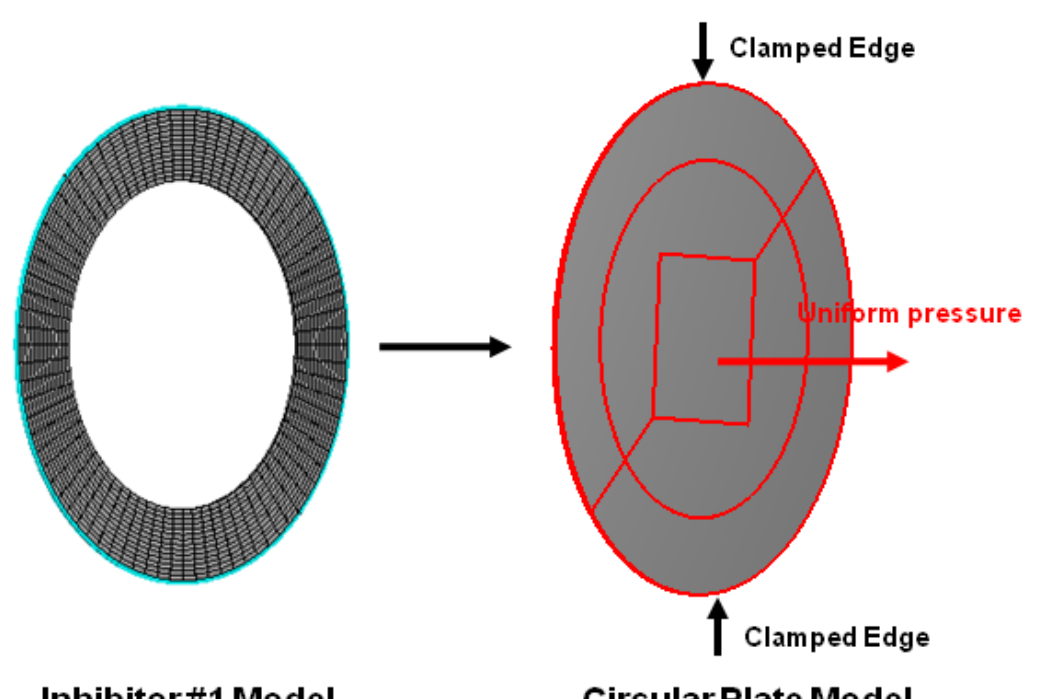

Circular Plate Model

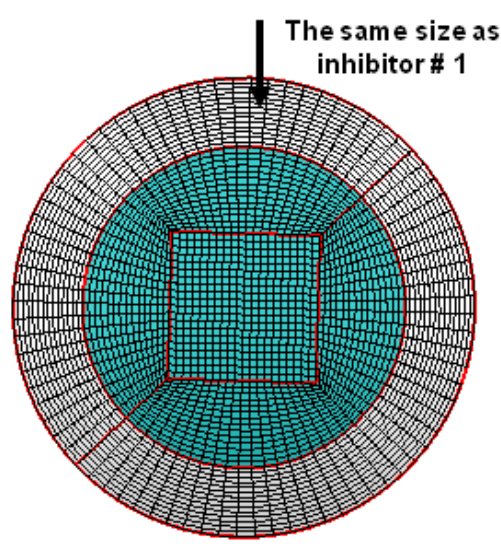

Circular Plate Grid

Figure 10.Verification model for circular plate under uniform pressure.

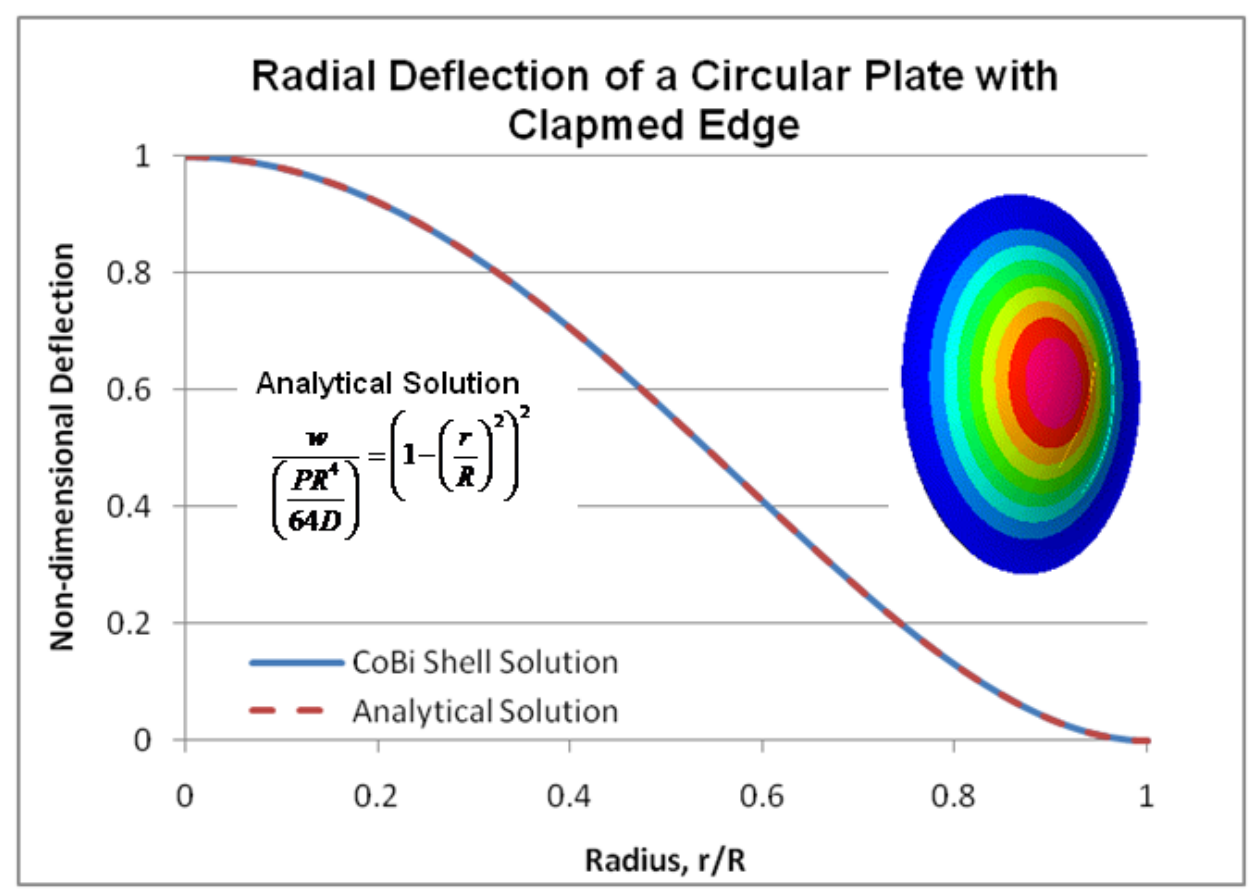

Figure 11.Comparison between analytical solution and present FEM solution from CoBi

To find the natural frequency of the above circular plate, the governing equation can be written as:

$$
\nabla^{4} w+\frac{m}{D} \frac{\partial^{2} w}{\partial t^{2}}=0
$$

where $\mathrm{m}$ is the plate mass per unit area. The eigenvalue solution of the above equation is in the form of Bessel functions:

$$
J_{0}(\gamma R) I_{1}(\gamma R)+J_{1}(\gamma R) I_{0}(\gamma R)=0
$$


The first few natural frequencies are [16]:

$$
\omega_{1}=\frac{k_{n}}{R^{2}} \sqrt{\frac{D}{m}} ; \mathrm{k}_{1}=10.22 ; \mathrm{k}_{2}=21.26 ; \mathrm{k}_{3}=34.88 ; \mathrm{k}_{4}=39.77
$$

Figure 12 shows the first few modal shapes and frequencies of the circular plate. The analytical solutions for the first 4 modes are compared with the present prediction. Excellent agreements are obtained for different modes.The results from NASTRAN are also shown in the same table. One can see that CoBi gives a better accuracy.

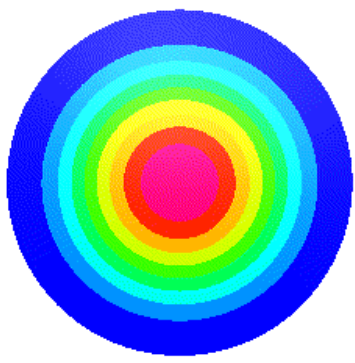

$1^{\text {st }}$ mode

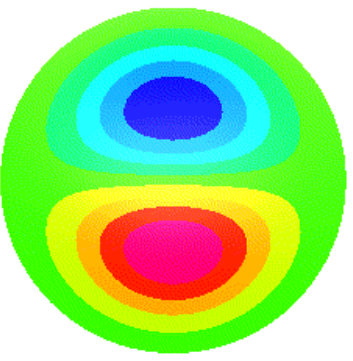

$2^{\text {nd }}$ mode

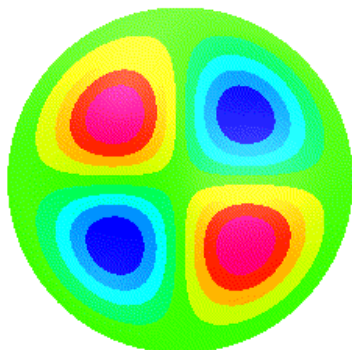

$3^{\text {rd }}$ mode

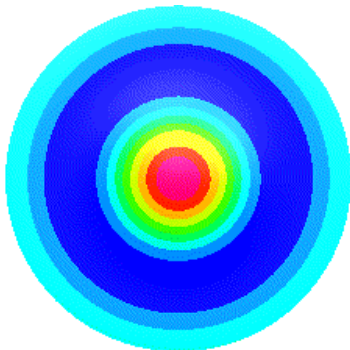

$4^{\text {th }}$ mode

Table 2. Modal Frequency Comparison

\begin{tabular}{|c|c|c|c|c|c|}
\hline \multicolumn{1}{|c|}{ Modes } & Analytical (Hz) & $\begin{array}{c}\text { NASTRAN } \\
\text { (Shell) }\end{array}$ & $\begin{array}{c}\text { CoBi } \\
\text { (Shell) }\end{array}$ & $\begin{array}{c}\text { NASTRAN } \\
\text { (Shell) }\end{array}$ & $\begin{array}{c}\text { CoBi } \\
\text { (Shell) }\end{array}$ \\
\hline $\begin{array}{c}\mathbf{1}^{\text {st }} \text { mode } \\
\text { (fundamental) }\end{array}$ & 1.867 & 1.888 & 1.866 & $-0.13 \%$ & $0.033 \%$ \\
$\begin{array}{c}2^{\text {nd }} \text { mode } \\
\text { (one nodal diameter) }\end{array}$ & 3.883 & 3.928 & 3.866 & $-0.17 \%$ & $-0.077 \%$ \\
$\begin{array}{c}3^{\text {rd }} \text { mode } \\
\text { (two nodal diameters) }\end{array}$ & 6.371 & 6.435 & 6.374 & $-0.46 \%$ & $-0.053 \%$ \\
$\begin{array}{c}4^{\text {th }} \text { mode } \\
\text { (one nodal circle) }\end{array}$ & 7.264 & 7.347 & 7.272 & $-0.26 \%$ & $-0.128 \%$ \\
\hline
\end{tabular}

Figure 12.Bending modes of circular plate and comparison with analytical solution and prediction by NASTRAN.

It should be noted that this verification exercise was conducted with the stand-alone CoBi binary constructed from the same source code as is coupled with the Loci/CHEM CFD program for the FSI simulation.

With the above successful verification study, a modal analysis was performed on the first 40 structural modes of the three inhibitors. The computed modal shapes for all three inhibitors are shown in Figure 13.As one can see, the first inhibitor has high exposed area to the fluid and hence has the lowest natural frequency of $7.62 \mathrm{~Hz}$. This mode is axi-symmetric. The mode number 2 and number 3 are a pair and have the same frequency with a modal shape rotated by $90 \mathrm{deg}$ to the axial direction. The $2^{\text {nd }}$ bending mode of the first inhibitor is mode number 37 and has a frequency of $45.2 \mathrm{~Hz}$. The first bending frequency for the second inhibitor is $30.55 \mathrm{~Hz}$, and the first bending model for the third inhibitor is beyond the first 40 modes of the present analysis. 


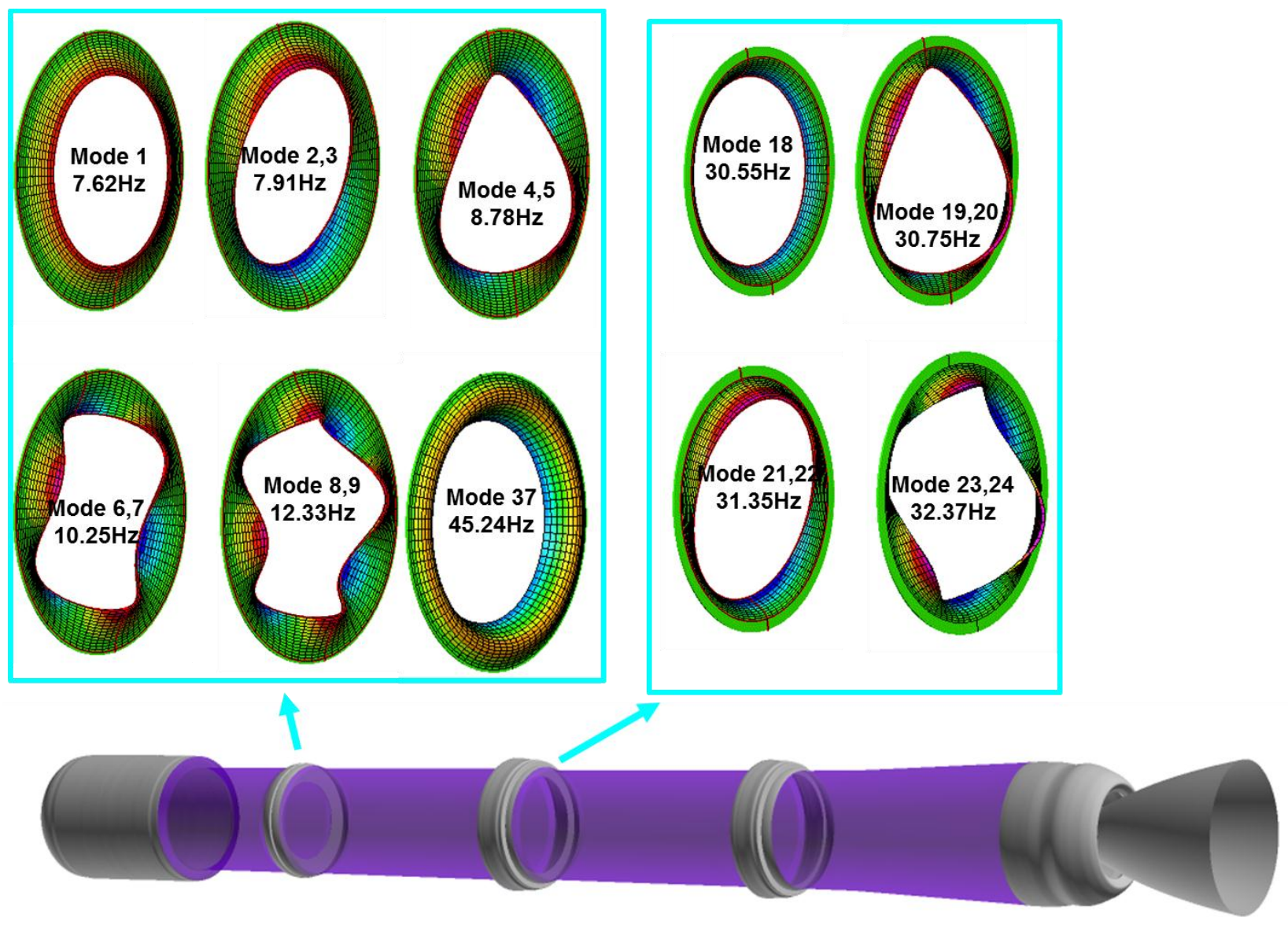

Figure 13.Structural modes for first two RSRM inhibitors computed using CoBi modal analysis.

\section{Coupling and Iteration Process}

A schematic is shown in Figure 14 to illustrate the iterative workflow of the tightly-coupled fluid-structure interaction process. The flow field is restarted from the previous $1^{\text {st }}$ order solution (but changed to $2^{\text {nd }}$ order in both time and space), and the structural solution is started with no deformation and zero velocity initial conditions. First the structural solver receives the pressure force acting on the structural boundary and then solves for the deformation. This deformation is then mapped to the surface mesh of the fluid mesh. The moving mesh deformation is activated to deform the volumetric fluid mesh based on the surface deformation. With this new deformation, the fluid field is solved to obtain a new pressure. This pressure is then feed to the structural solver again. The fluid and structure are solved in this tightly-coupled manner at every sub-iteration within each time step until convergence is reached in each solver. Typically, residual from structural solver drops 3 orders of magnitude within 6 sub-iterations as shown in Figure 15. 


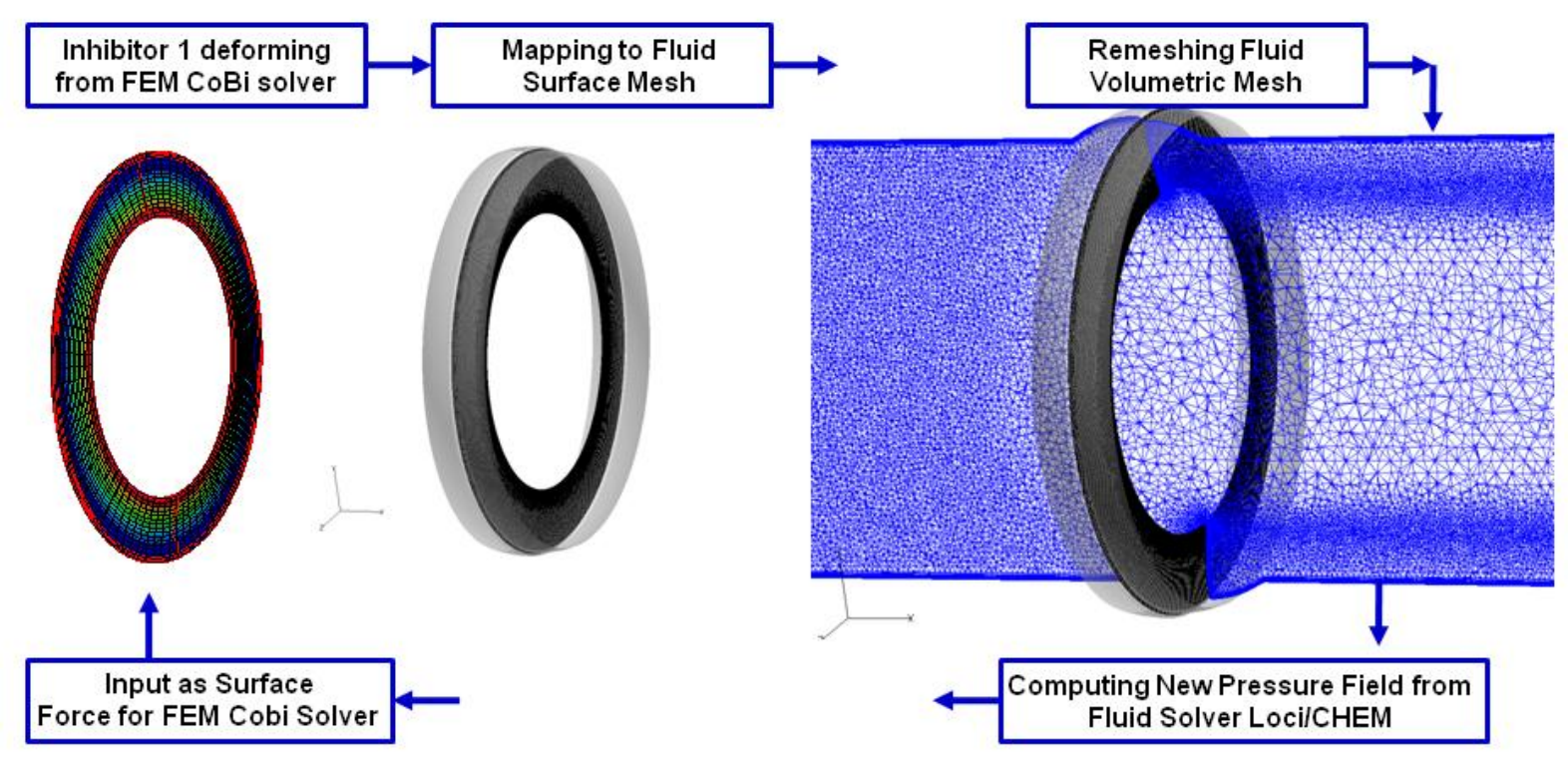

Figure 14.Schematic showing iterative workflow of tightly-coupled fluid-structure interaction process.

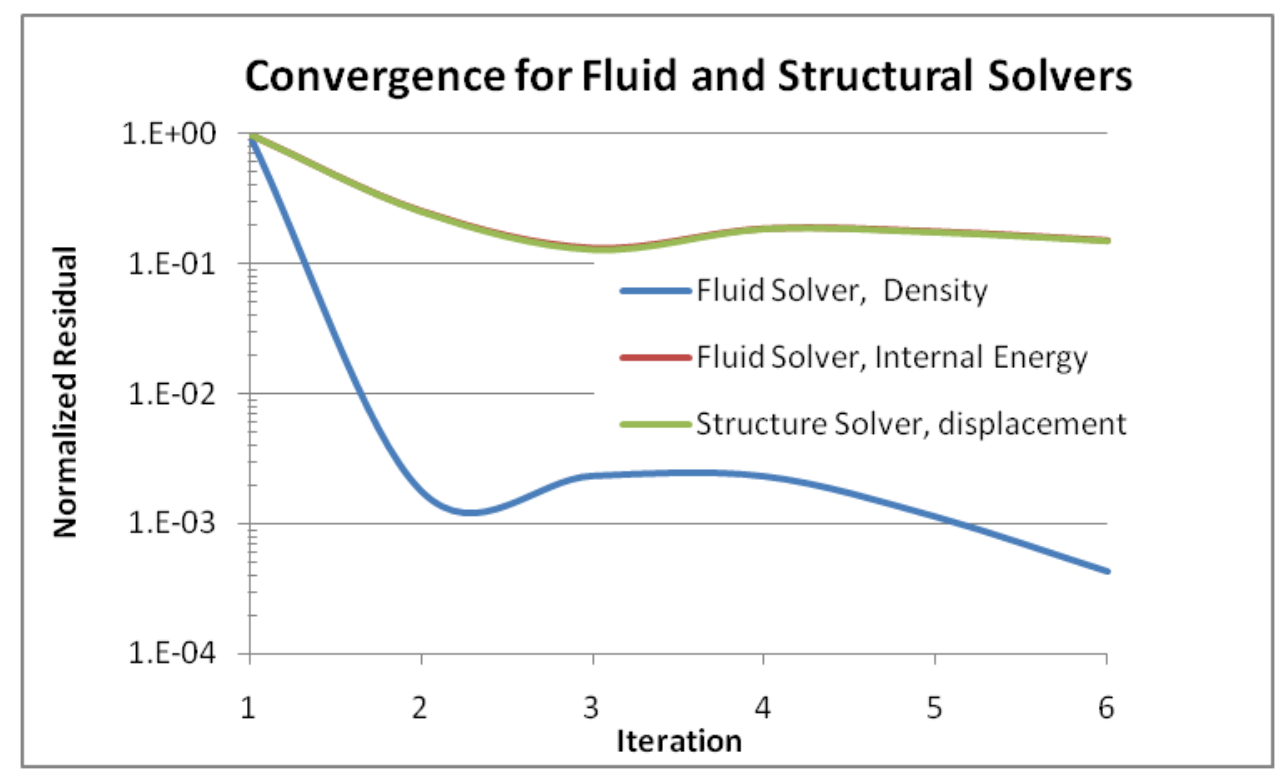

Figure 15.Normalized residual drops for fluid and structural solvers during a typical time step

\section{Coupled Fluid-Structural Solution}

Tightly-coupled fluid-structural interaction simulations were carried out for the RSRM application with flexible inhibitors until $t=0.60 \mathrm{~s}$. The instantaneously computed vorticity field on a slice through the RSRM, including the deforming solid surfaces, at six different time instances from $0.1 \mathrm{~s}$ to $0.6 \mathrm{~s}$ in even $0.1 \mathrm{~s}$ increments is shown in Figure 16. Unsteady vortex shedding is clearly observed at each of the flexible inhibitors, and the first inhibitor is undergoing very large deformations in response to the large pressure gradients present within the solid rocket motor. 


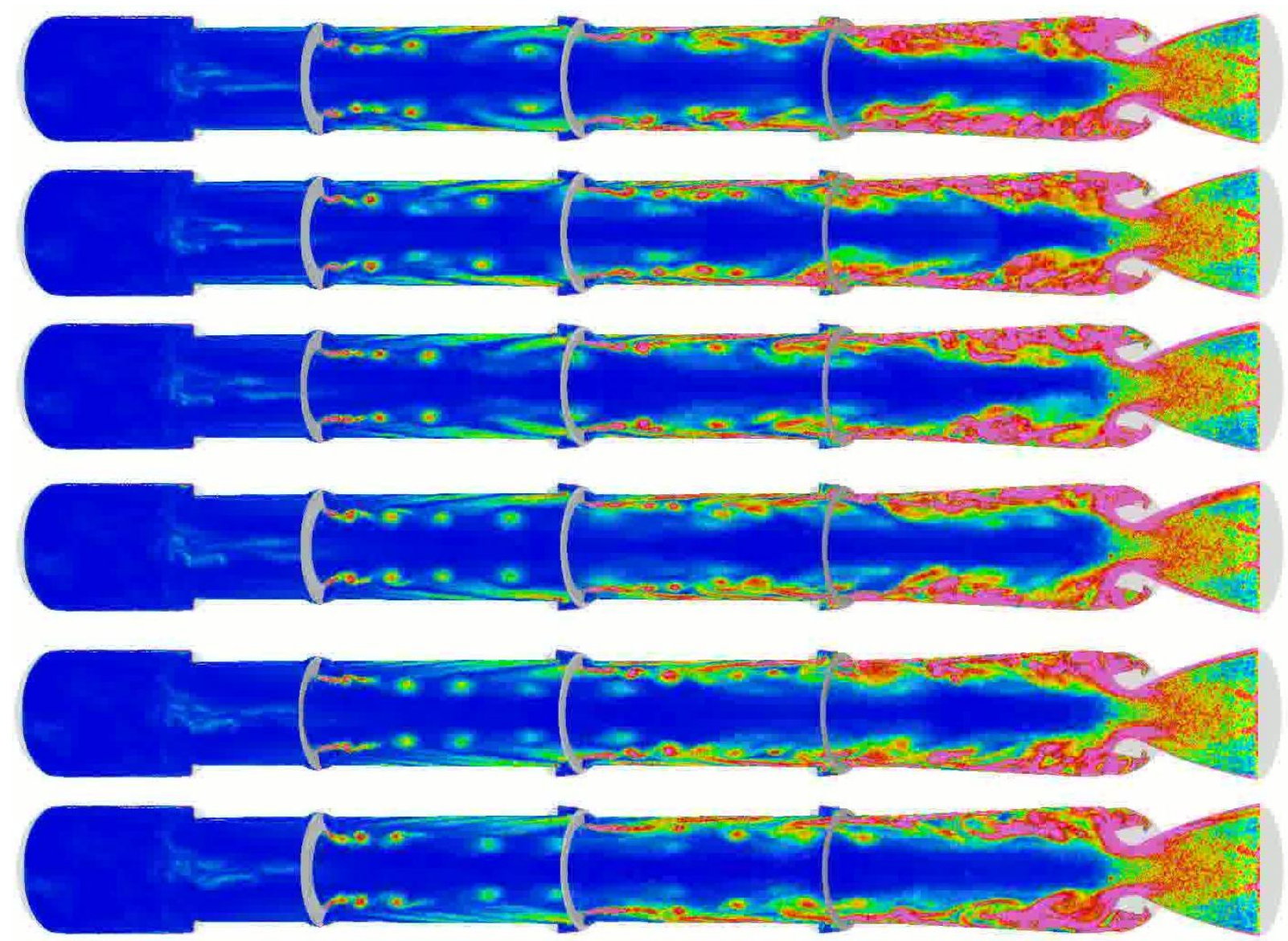

Figure 16.Instantaneous vorticity field for tightly-coupled FSI simulation of RSRM with flexible inhibitors: From 0.1s (Top) to 0.6s (Bottom) in even 0.1s increments.

The large structural deformations are very clearly observed in the close-up views of the first inhibitor along with the fluid mesh colored by vorticity presented in Figure 17. The inhibitor tip can be seen deflecting up to about 20-30 degrees in each direction in response to the unsteady flow with large pressure gradients.

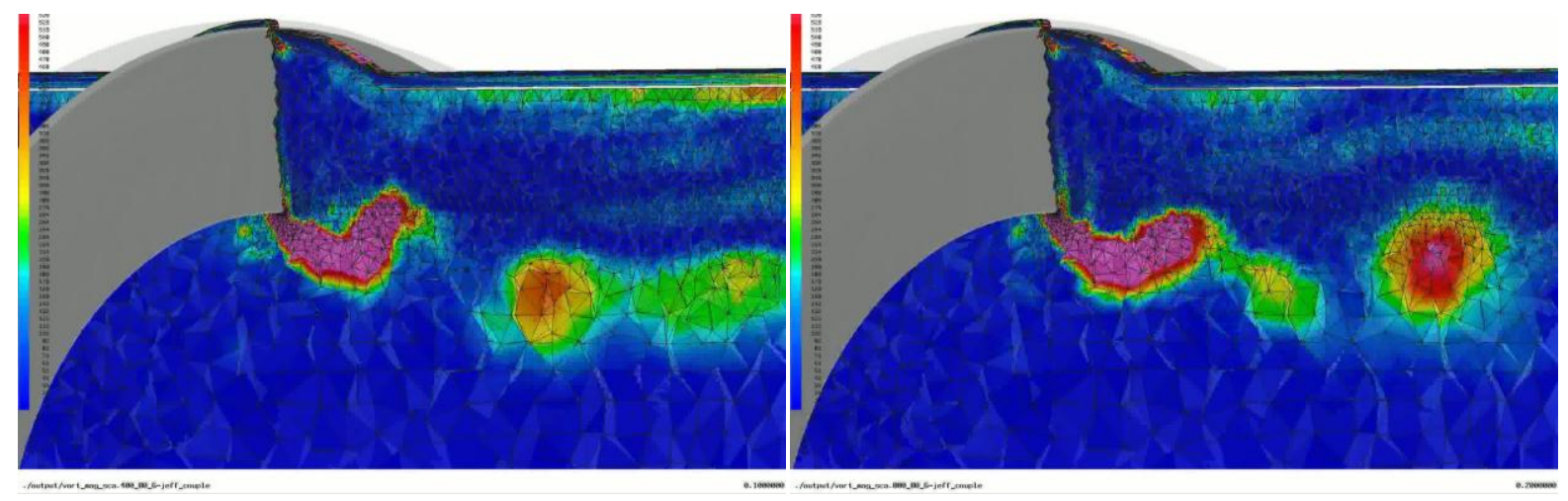



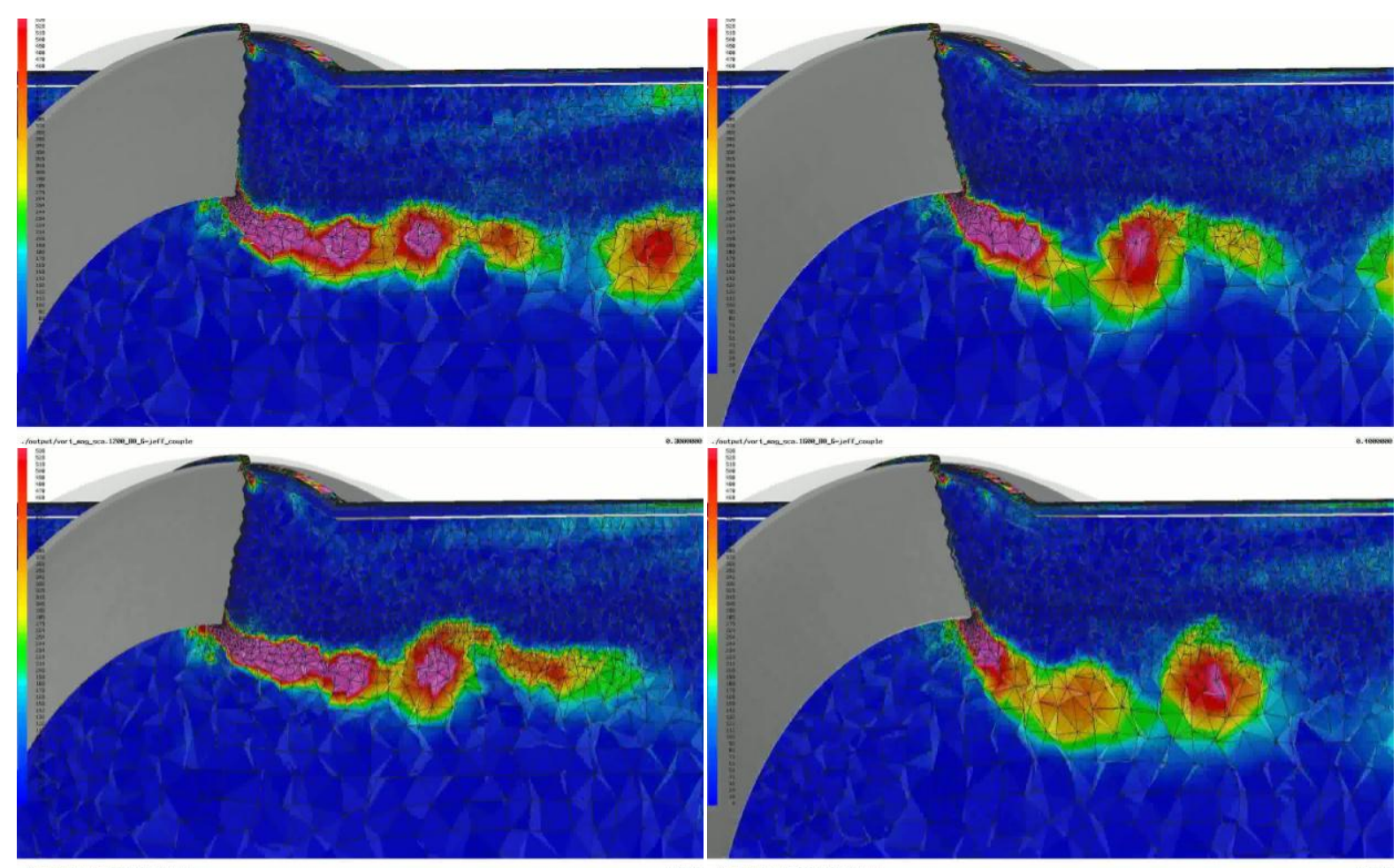

Figure 17.Close-up view of first inhibitor, fluid mesh and instantaneous vorticity field for tightly-coupled FSI simulation of RSRM with flexible inhibitors: From 0.1s (Top left) to 0.6s (Bottom right) in even 0.1s increments.

To show the three-dimensional nature of the unsteady vortical flow, instantaneous iso-surfaces of helicity at three different time instances $(0.05 \mathrm{~s}, 0.10 \mathrm{~s}$, and $0.15 \mathrm{~s})$ are presented in Figure 18 . Here the helicity is defined as the dot product of velocity vector with vorticity vector. In response to the periodic unsteady vortex shedding, vortex-vortex interactions, and vortex interactions with flexible inhibitors, the flow becomes increasingly helical as it travels downstream toward the nozzle exit.
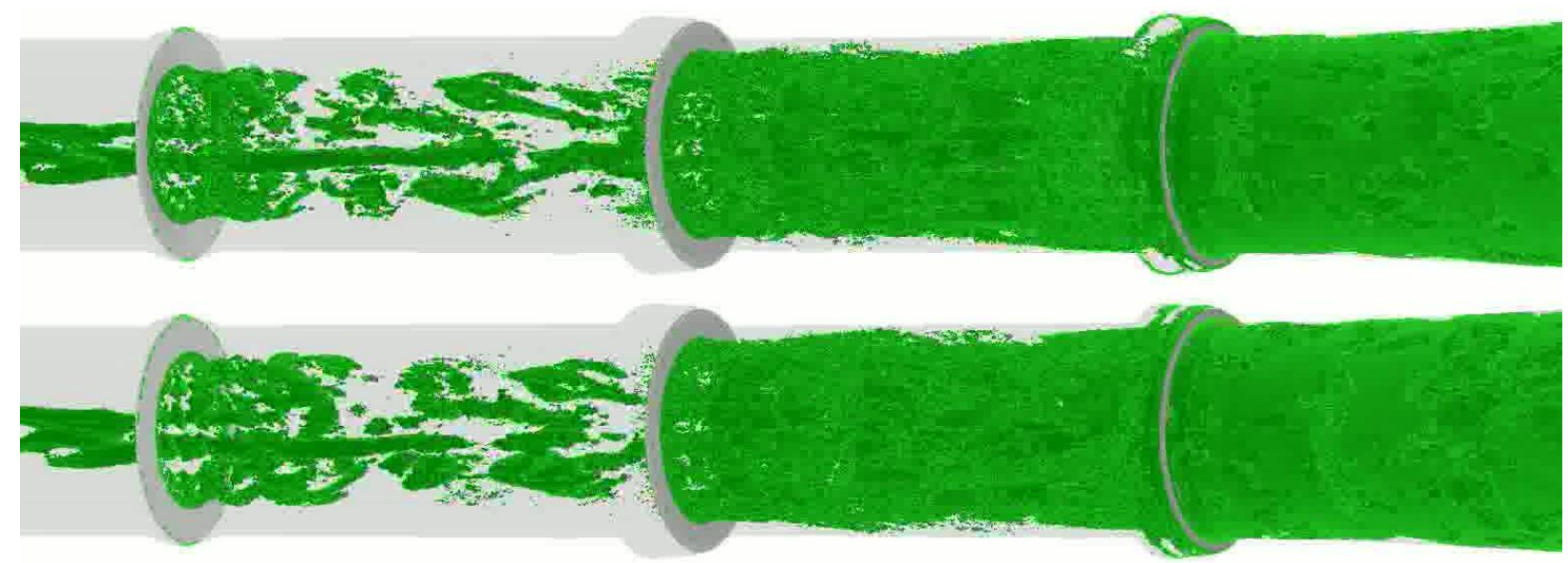


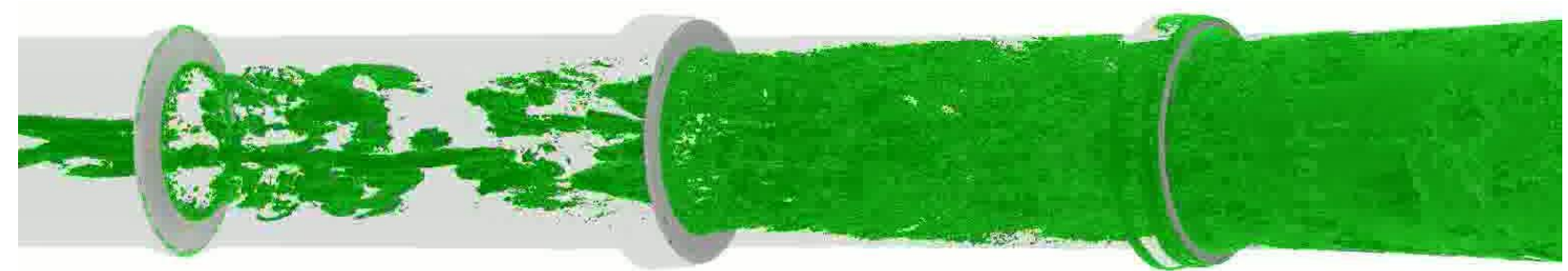

Figure 18.Instantaneous helicity field for tightly-coupled FSI simulation of RSRM with flexible inhibitors: (Top) 0.05s; (Middle) 0.10s; (Bottom) 0.15s;

Figure 19 displays the time history of the three inhibitor tip displacements from the coupled solution. Due to small extrusion into the flow field, the $2^{\text {nd }}$ and $3^{\text {rd }}$ inhibitors exhibit very small displacements in response to the flow. For the $3^{\text {rd }}$ inhibitor it behaves essentially as a rigid body. The $2^{\text {nd }}$ inhibitor shows periodic motion at its own first natural frequency of $30.55 \mathrm{~Hz}$ (Figure 11).
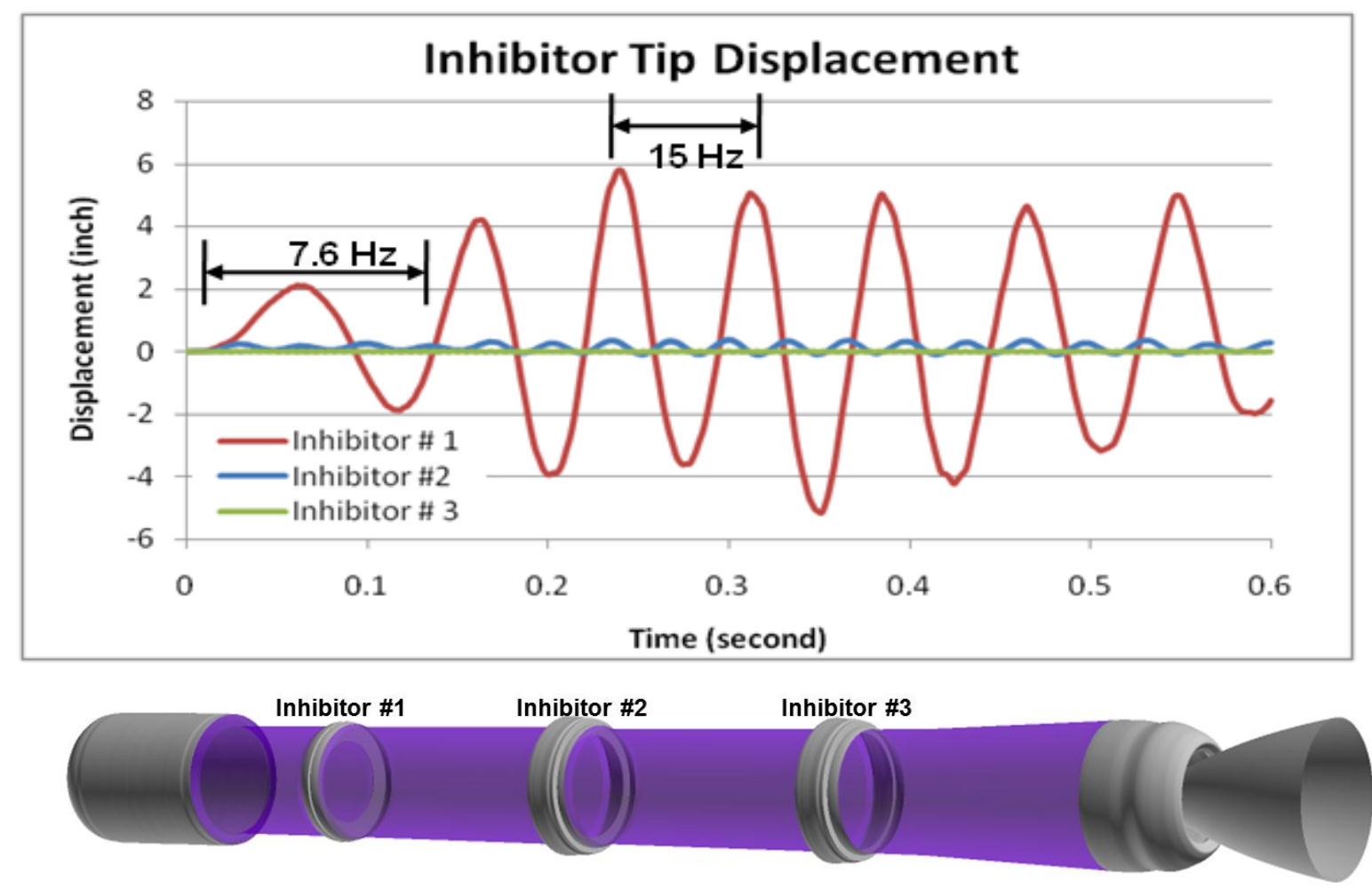

Figure 19.Time history of inhibitor tip displacement showing $1^{\text {st }}$ inhibitor shift from its own first modal frequency $(7.5 \mathrm{~Hz})$ to the $\mathrm{SRM}$ acoustic frequency $(15 \mathrm{~Hz})$.

Due to its flexibility, the $1^{\text {st }}$ inhibitor shows some very interesting dynamics. Initially, the $1^{\text {st }}$ inhibitor oscillates at its own natural frequency of $7.5 \mathrm{~Hz}$, but gradually shifts to the solid rocket motor acoustic frequency of $15 \mathrm{~Hz}$. Its motion is driven by the internal acoustic wave in the first mode, and the displacements appear to settle to a periodic motion. As shown in Figure 20, at $15 \mathrm{~Hz}$ the $1^{\text {st }}$ inhibitor vibrates at its own first modal shape, rather than its own modal shape at $15.2 \mathrm{~Hz}$. This implies that the driving force (or the pressure field) is axi-symmetric. When the inhibitor vibrates at the rocket motor first acoustic modal frequency, it will shed a coherent vortex at $15 \mathrm{~Hz}$. It will be interesting to determine its feedback on the acoustic wave amplitude. This will be investigated in future studies. 


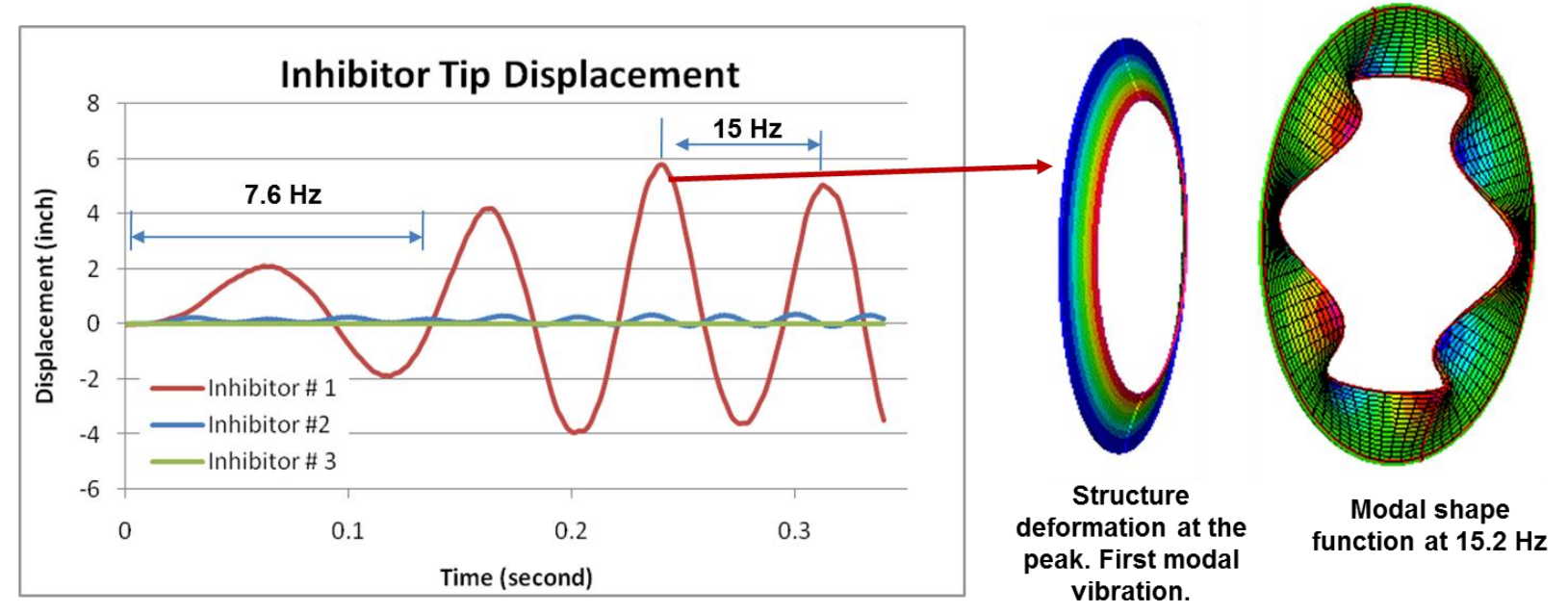

Figure 20.Comparison of structural deformation at the peak tip displacement for the first inhibitor with its computed modal shape at $15.2 \mathrm{~Hz}$.

\section{Summary and Potential Applications}

A new capability to fully couple a production CFD solver (Loci/CHEM) to a structural solver, has been demonstrated. Initial results for flexibility inhibitor in RSRM show a strong coupling of inhibitor dynamics with acoustic pressure oscillation inside RSRM. This new capability can provide insight to understand the thrust oscillation issues in SLS design.

\section{References}

${ }^{1}$ McWhorter, B. B., "Real-Time Inhibitor Recession Measurements in Two Space Shuttle Reusable Solid Rocket Motors", AIAA/ASME/SAE/ASEE Joint Propulsion Conference and Exhibit, AIAA-2003-5107, July 2-23, 2003.

${ }^{2}$ Plourde, F., Najjar, F. M., Vetel, J., Wasistho, B., Doan, K. S., and Balachandar, S., Numerical Simulations of Wall and Shear-Layer Instabilities in a Cold Flow Set-up, $37^{\text {th }}$ AIAA/ASME/SAE/ASEE Joint Propulsion Conference and Exhibit, AIAA-2003-4674, July 20-23 (2003).

${ }^{3}$ Anthoine, J., and Buchkin, J-M, Effect of Nozzle Cavity on Resonance in Large SRM: Numerical Simulations, J. Propulsion and Power, 19 (3), (2003), pp. 374-384.

${ }^{4}$ Mason, D. R., Morstaadt, R. A., Cannon, S. M., Gross, E. G., and Nielson, D. B., Pressure Oscillations and Structural Vibrations in Space Shuttle RSRM and ETM-3 Motors, 38th AIAA/ASME/SAE/ASEE Joint Propulsion Conference and Exhibit, AIAA-2004-3898, July 11-14, (2004).

${ }^{5}$ Mastrangelo, et al., "Segmented SRM Pressure Oscillation Demonstrator", AIAA/ASME/SAE/ASEE $47^{\text {th }}$ Joint Propulsion Conference, 2011. AIAA-2011-6056, 2011.

${ }^{6}$ Roach, R. L., Gramoll, K. C., Weaver, M. A, and Flandro, G. A., "Fluid-Structure Interaction of Solid Rocket Motor Inhibitors", 28th AIAA/ASME/SAE/ASEE Joint Propulsion Conference and Exhibit, AIAA-92-3677, July 68 (1992).

${ }^{7}$ Weaver, M. A., Gramoll, K. C., and Roach, R. L., "Structural Analysis of a Flexible Structural Member Protruding into an Interior Flow Field", 34th AIAA/ASME/ASCE/AHS/ASC Structures, Structural Dynamics, and Materials Conference, AIAA-93-1446, (1993).

${ }^{8}$ Fiedler, R., Namazifard, A., Campbell, M., and Xu, F., "Detailed Simulations of Propellant Slumping in the Titan IV SRMUPQM-1,"42nd AIAA/ASME/SAE/ASEE Joint propulsion Conference and Exhibit, Sacramento, CA, AIAA Paper 06-4592, July 2006.

${ }^{9}$ Wasistho, B., Fiedler, R., Namazifard, A., and Mclay, C., "Numerical Study of Turbulent Flow in SRM with

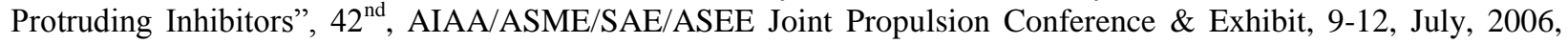
Sacramento, CA., AIAA-2006-4589, 2006.

${ }^{10}$ R. E. Harris and E. Luke, "Towards an Integrated Fluid-Structure-Thermal Simulation Capability in the Loci/CHEM Solver”, 51thAIAA Aerospace Science Meeting, 7-10 January 2013, Grapevine, TX, AIAA-2013-0099. 
${ }^{11}$ Luke, E. and George, T., "Loci: “A Rule-Based Framework for Parallel Multidisciplinary Simulation Synthesis," Journal of Functional Programming, Special Issue on Functional Approaches to High-Performance Parallel Programming, Vol. 15, No.3, 2005, pp. 477-502.

${ }^{12}$ Luke, E., "On Robust and Accurate Arbitrary Polytope CFD Solvers (Invited),"AIAA 2007-3956, AIAA Computational Fluid Dynamics Conference, Miami, 2007.

${ }^{13}$ Liu, Q., Luke, E., and Cinnella, P., "Coupling Heat Transfer and Fluid Flow Solvers for Multi-Disciplinary Simulations," AIAA Journal of Thermophysics and Heat Transfer, Vol. 19, No. 4, 2005, pp 417-427.

${ }^{14}$ Roy, C., Tendean, E., Veluri, S.P., Rifki, R., Luke, E., and Hebert, S., "Verification of RANS Turbulence Models in Loci-CHEM using the Method of Manufactured Solutions", 18th AIAA Computational Fluid Dynamics Conference, 25-28 June, 2007, Miami, FL. AIAA-2007-4203.

${ }^{15}$ Davis, P., Tucker, P. K., and Kenny, R. J., "RSSM Thrust Oscillations Simulations", Presented at the JANNAF 2011 MSS/LPS/SPS Joint Subcommittee Meeting, Huntsville, AL, 12/08/2011.

${ }^{16}$ Rilkey, W.D., Formulas for Stress, Strain, and Structural Matrices, John Wiley and Sons, Inc., 1994. ISBN 0471527467

${ }^{17}$ Yang, H. Q and West, J. "Computational Fluid Dynamics Analysis of J-2X LOX Inducer Modal Damping”, ER42(12-097)/ESTSG-FY12-623, 2012. 